\title{
SECOND-ORDER EFFICIENT OPTIMALITY CONDITIONS FOR SET-VALUED VECTOR OPTIMIZATION IN TERMS OF ASYMPTOTIC CONTINGENT EPIDERIVATIVES *
}

\author{
NGuyen Minh Tung**
}

\begin{abstract}
We propose a generalized second-order asymptotic contingent epiderivative of a set-valued mapping, study its properties, as well as relations to some second-order contingent epiderivatives, and sufficient conditions for its existence. Then, using these epiderivatives, we investigate set-valued optimization problems with generalized inequality constraints. Both second-order necessary conditions and sufficient conditions for optimality of the Karush-Kuhn-Tucker type are established under the second-order constraint qualification. An application to Mond-Weir and Wolfe duality schemes is also presented. Some remarks and examples are provided to illustrate our results.
\end{abstract}

Mathematics Subject Classification. 90C26, 90C46, 90C48.

Received July 21, 2020. Accepted March 10, 2021.

\section{INTRODUCTION}

Optimality conditions play an important role in both theory and numerical methods in optimization. First-order conditions have been developed for many decades and now the research outcomes are very rich. Second-order optimality conditions have also attracted strong attention since they refine the first-order ones by second-order information and are much helpful to recognize optimal solutions as well as to design numerical algorithms for computing them. For second-order conditions for (scalar and vector) single-valued problems, see, e.g. $[8,13,20,21,24,26,27,35]$ and the references therein.

In the last several decades, set-valued optimization problems have become more attractive because they can be applicable for a wide range of practical situations and many advanced mathematical tools can be invoked for the study. Many generalized derivatives have been proposed and applied to investigate optimality conditions for nonsmooth problems. There are two main types of optimality conditions. Conditions of the primal form are expressed as the disjointedness of sets approximating the data of the optimization problem and sets of "better" directions of the objective and feasible directions of the constraints. Conditions of the dual form are represented by multiplier rules of the Fritz John or Karush-Kuhn-Tucker (KKT) type. For first-order considerations, Aubin [2] proposed a concept of a contingent derivative for set-valued maps, which was used to establish Fritz John multiplier rules in Corley [9] and followed by many papers. Jahn and Rauh [18] introduced

Keywords. Asymptotic contingent epiderivative, optimality conditions, Karush-Kuhn-Tucker multiplier, constraint qualification, duality.

* DEDICATED TO PROFESSOR PHAN QUOC KHANH ON THE OCCASION OF HIS 75TH BIRTHDAY.

Faculty of Mathematical Economics, Banking University of Ho Chi Minh City, Ho Chi Minh City, Vietnam.

** Corresponding author: tungnm@buh.edu.vn 
a notion of a contingent epiderivative, which is an extension of the directional derivative, but it is a single map and its existence still remains a difficult problem. To overcome this difficulty, Chen and Jahn [6] proposed a generalized contingent epiderivative of a set-valued map and applied it to obtain primal-form optimality conditions in [6,18] and KKT multiplier rules in [17]. Since minimization is more closely related to epigraphs than to graphs, these types of epiderivatives have advantages over the corresponding types of derivatives based on graphs. By using contingent epiderivatives, Götz and Jahn in [12] obtained a first-order Karush-KuhnTucker (KKT) necessary optimality condition. With the well-known Dubovitski-Milutin approach, Issac and Khan in [15] established a multiplier rule for set-valued optimization with generalized inequality constraints. For the first-order optimality conditions, another fruitful approach in set-valued optimization is the dual space approach initiated by Mordukhovich (see [34,45]).

The second-order optimality conditions for set-valued optimization take in the literature a place much smaller than that of the first-order ones. Let us mention the results related to the present paper. In [11], Durea employed second-order contingent derivatives to establish primal-form conditions. In [19], Jahn et al. proposed a notion of generalized second-order contingent epiderivative and applied it to obtain primal-form optimality conditions for problems subject to geometric constraints (set constraints). It is worth noting that a second-order contingent set is in general not a cone and nonconvex. Therefore the corresponding second-order contingent derivative and epiderivative are not convenient tools in some cases. To improve the situation, by using a second-order composed set, Li et al. proposed a second-order composed contingent epiderivative in [32] and a generalized second-order composed contingent derivative in [46] and applied them to problems with set constraints in [32] to get primal-form conditions and to problems with generalized inequality constraints in [46] to obtain KKT rules. By using the second-order contingent derivative, Khanh et al. [28] established some second-order KKK conditions for a set-valued optimization problem subject to generalized inequality constraints. In terms of second-order asymptotic contingent derivatives, in [25] Khan and Tammer proved second-order multiplier rules, following the Dubovitskii-Milutin approach. As far as we know, very few papers apply asymptotic derivatives in set-valued optimization.

Motivated by the above observations, in this paper by using second-order asymptotic contingent cones, we propose a concept of a generalized second-order asymptotic contingent epiderivative for a set-valued mapping. We discuss its properties as well as its relations to known generalized second-order contingent epiderivatives. Under standard assumptions, sufficient conditions are established for the existence of generalized second-order asymptotic contingent epiderivatives. Furthermore, by employing the free disposal condition, another existence theorem is also obtained. Using generalized second-order asymptotic contingent epiderivatives, we acquire second-order KKT multiplier rules under qualification conditions of the Kurcyusz-Robinson-Zowe (KRZ) type for a set-valued optimization problem subjected to a generalized inequality constraints, and compare these qualification conditions with some other existing ones. Note that dual-form optimality conditions expressed by multiplier rules are more useful than primal-form conditions, specially in applications and numerical methods. Moreover, KKT multiplier rules are more significant than Fritz John ones because they contain explicitely nonzero multipliers of the objectives and hence express optimality properties, not only extremal properties of the constraints as Fritz John rules do when the objective multiplier is zero. Note also that our KKT multiplier rules contain the envelope-like effect because the feasible set includes critical directions. This phenomenon was discovered by Kawasaki in [24], and developed in [8,21,35] for $C^{2}$ scalar programming, in [13,26] for nonsmooth multiobjective programming, in [27] for infinite dimensional nonsmooth optimization, and in [28-30] for set-valued optimization.

Beside optimality condition theory, duality theory also occupies an important place in the center of optimization theory. In [44], Weir and Mond proved duality results for weak minimizer of multiple objective optimization problems under pseudo-convexity and quasi-convexity assumptions. Sach et al. [37] used the codifferential of set-valued mappings to discuss Mond-Weir type and Wolfe type weak duality and strong duality results of set-valued optimization problems under generalized invexity. By using the higher-order contingent derivatives, Li et al. [31] proposed a higher-order Mond-Weir dual for a set-valued optimization problem and obtained their weak, strong, and converse duality properties. By virtue of higher order weak adjacent contingent epiderivatives 
and Henig efficiency, Chen et al. [7] studied a higher order Mond-Weir type dual problem and a higher order Wolfe type dual problem for a constrained set-valued optimization problem and obtained the corresponding duality properties. Sun et al. [42] proposed a robust-type subdifferential constraint qualification for a uncertain convex optimization problem. Then they applied them to investigate Wolfe type robust duality between the uncertain convex optimization problem and its uncertain dual problem. Recently, in $[39,42]$, the authors studied some new characterizations of robust optimal solution sets for an uncertain optimization problems with a new Lagrangian dual approach. Inspired by the work of $[1,7,31,39,42,44]$, we apply these preceding results to study a Mond-Weir type dual problem and a Wolfe type dual problem for the set-valued optimization problem, and discuss the corresponding weak duality, strong duality and converse duality properties.

The organization of the paper is as follows. In Section 2, we collect definitions and preliminary facts for our use in the sequel. Section 3 contains some properties of generalized second-order asymptotic contingent epiderivatives. Section 4 is devoted to second-order necessary conditions and sufficient conditions in terms of KKT multipliers. Section 5 is an application of the results obtained in Sections 3 and 4 to duality.

\section{Preliminaries}

Let $X, Y$ and $Z$ be real Banach spaces, $\mathbb{N}, \mathbb{R}^{n}$ and $\mathbb{R}_{+}^{n}$ be the set of the natural numbers, a $n$-dimensional Banach space and its nonnegative orthant, respectively (resp). $B_{X}$ denotes the open unit ball of $X$ and $B_{X}(x, r)$ the open ball of center $x$ and radius $r>0$. For $M \subseteq X$, int $M, \mathrm{cl} M$, and bd $M$ stand for its interior, closure and boundary, resp, of $M$. The cone generated by $M$ is cone $M:=\{\lambda x \mid \lambda \geq 0, x \in M\}$. For a set-valued map $\Phi: X \rightrightarrows Y$, the domain, graph, and epigraph of $\Phi$ are defined as

$$
\begin{gathered}
\operatorname{dom} \Phi:=\{x \in X \mid \Phi(x) \neq \emptyset\}, \quad \operatorname{gph} \Phi:=\{(x, y) \in X \times Y \mid y \in \Phi(x)\} \\
\operatorname{epi} \Phi:=\{(x, y) \in X \times Y \mid y \in \Phi(x)+C\} .
\end{gathered}
$$

For a subset $S \subseteq X, \Phi(S):=\bigcup_{x \in S} \Phi(x)$ and the profile (or epigraphical) map $\Phi_{+}: X \rightrightarrows Y$ is defined by $\Phi_{+}(x):=\Phi(x)+C . \Phi$ is said to be $C$-convex if, for all $x_{1}, x_{2} \in X$ and $\lambda \in[0,1]$,

$$
\lambda \Phi\left(x_{1}\right)+(1-\lambda) \Phi\left(x_{2}\right) \subseteq \Phi\left(\lambda x_{1}+(1-\lambda) x_{2}\right)+C .
$$

It is easy to verify that $\Phi$ is $C$-convex if and only if epi $\Phi$ is a convex set in $X \times Y$. $\Phi$ is said to be $C$-subadditive if, for all $x_{1}, x_{2} \in X, \Phi\left(x_{1}\right)+\Phi\left(x_{2}\right) \subseteq \Phi\left(x_{1}+x_{2}\right)+C$.

Definition 2.1 (see [16]). Let $Y$ be partially ordered by a pointed, closed and convex cone $C \subseteq Y, \Delta$ be a nonempty subset of $Y$, and a point $\bar{y} \in \Delta$.

(i) $\bar{y}$ is called a minimal element of $\Delta$ if $(\{\bar{y}\}-C \backslash\{0\}) \cap \Delta=\emptyset$.

(ii) Supposing int $C \neq \emptyset, \bar{y}$ is called a weak minimal element of $\Delta$ if $(\{y\}-\operatorname{int} C) \cap \Delta=\emptyset$.

The set of all the minimal elements (resp, weak minimal elements) of $\Delta$ is denoted by $\operatorname{Min}_{C} \Delta$ (resp, $\mathrm{WMin}_{C}$ $\Delta)$. It is obvious that $\operatorname{Min}_{C} \Delta \subseteq \mathrm{WMin}_{C} \Delta$.

Definition 2.2 (see $[16,33]$ ). Let $Y$ be partially ordered by a pointed, closed and convex cone $C \subseteq Y$, and $\Delta$ be a nonempty subset of $Y$.

(i) A sequence $\left\{y_{n}\right\} \subseteq Y$ is said to be $C$-decreasing if $y_{j} \leq_{C} y_{i}$ for all $i, j \in \mathbb{N}$ with $i \leq j$.

(ii) $\Delta$ is said to be $C$-lower bounded if there exists a $y \in Y$, such that $\Delta \subseteq\{y\}+C$.

(iii) $\Delta$ is said to be have the domination property if $\Delta \subseteq \operatorname{Min}_{C} \Delta+C$.

(iv) The convex cone $C$ is said to be Daniel if every $C$-decreasing and $C$-lower bounded sequence in $Y$ converges to its infimum.

The following tangent cones and second-order tangent sets will be used in this paper. 
Definition 2.3 (see $[8,20,35]$ ). Let $M \subseteq X$ and $x_{0}, u \in X$.

(i) The contingent cone (resp, adjacent cone and interior cone) of $M$ at $x_{0}$ is

$$
\begin{aligned}
T\left(M, x_{0}\right) & :=\left\{u \in X \mid \exists t_{n} \downarrow 0, \exists u_{n} \rightarrow u, \forall n \in \mathbb{N}, x_{0}+t_{n} u_{n} \in M\right\} \\
\left(T^{b}\left(M, x_{0}\right)\right. & :=\left\{u \in X \mid \forall t_{n} \downarrow 0, \exists u_{n} \rightarrow u, \forall n \text { large, } x_{0}+t_{n} u_{n} \in M\right\}, \\
I T\left(M, x_{0}\right) & \left.:=\left\{u \in X \mid \forall t_{n} \downarrow 0, \forall u_{n} \rightarrow u, \forall n \text { large, } x_{0}+t_{n} u_{n} \in M\right\}\right) .
\end{aligned}
$$

(ii) The second-order contingent set (resp, adjacent set and interior set) of $M$ at $x_{0}$ in direction $u$ is

$$
\begin{aligned}
T^{2}\left(M, x_{0}, u\right) & :=\left\{w \in X \mid \exists t_{n} \downarrow 0, \exists w_{n} \rightarrow w, \forall n \in \mathbb{N}, x_{0}+t_{n} u+\frac{1}{2} t_{n}^{2} w_{n} \in M\right\} \\
\left(A^{2}\left(M, x_{0}, u\right)\right. & :=\left\{w \in X \mid \forall t_{n} \downarrow 0, \exists w_{n} \rightarrow w, \forall n \text { large, } x_{0}+t_{n} u+\frac{1}{2} t_{n}^{2} w_{n} \in M\right\}, \\
I T^{2}\left(M, x_{0}, u\right) & \left.:=\left\{w \in X \mid \forall t_{n} \downarrow 0, \forall w_{n} \rightarrow w, \forall n \text { large }, x_{0}+t_{n} u+\frac{1}{2} t_{n}^{2} w_{n} \in M\right\}\right) .
\end{aligned}
$$

(iii) The asymptotic second-order tangent cone (resp, adjacent cone and interior cone) of $M$ at $x_{0}$ in direction $u$ is

$$
\begin{aligned}
T^{\prime \prime}\left(M, x_{0}, u\right) & :=\left\{w \in X \mid \exists\left(t_{n}, r_{n}\right) \downarrow(0,0): t_{n} r_{n}^{-1} \downarrow 0, \exists w_{n} \rightarrow w, \forall n \in \mathbb{N}, x_{0}+t_{n} u+\frac{1}{2} t_{n} r_{n} w_{n} \in M\right\} \\
\left(A^{\prime \prime}\left(M, x_{0}, u\right)\right. & :=\left\{w \in X \mid \forall\left(t_{n}, r_{n}\right) \downarrow(0,0): t_{n} r_{n}^{-1} \downarrow 0, \exists w_{n} \rightarrow w, \forall n \text { large, } x_{0}+t_{n} u+\frac{1}{2} t_{n} r_{n} w_{n} \in M\right\}, \\
I T^{\prime \prime}\left(M, x_{0}, u\right) & \left.:=\left\{w \in X \mid \forall\left(t_{n}, r_{n}\right) \downarrow(0,0): t_{n} r_{n}^{-1} \downarrow 0, \forall w_{n} \rightarrow w, \forall n \text { large, } x_{0}+t_{n} u+\frac{1}{2} t_{n} r_{n} w_{n} \in M\right\}\right) .
\end{aligned}
$$

$M \subseteq X$ is called second-order derivable (resp, asymptotic derivable) at $\left(x_{0}, u\right)$ if

$$
T^{2}\left(M, x_{0}, u\right)=A^{2}\left(M, x_{0}, u\right)\left(\operatorname{resp}, T^{\prime \prime}\left(M, x_{0}, u\right)=A^{\prime \prime}\left(M, x_{0}, u\right)\right) .
$$

Note that, if $x_{0} \notin \mathrm{cl} M$, then all the above tangent sets are empty; and if $u \notin T\left(M, x_{0}\right)$, then all the secondorder tangent sets are empty. Hence, the conditions such as $x_{0} \in \operatorname{cl} M$ and $u \in T\left(M, x_{0}\right)$ are always assumed. $T^{2}\left(M, x_{0}, u\right), T^{\prime \prime}\left(M, x_{0}, u\right)$ and $T\left(T\left(M, x_{0}\right), u\right)$ are closed sets contained in clcone[cone $\left.\left.\left(M-x_{0}\right)-u\right)\right] . T\left(S, x_{0}\right)$, $T\left(T\left(M, x_{0}\right), u\right), T^{\prime \prime}\left(M, x_{0}, u\right), A^{\prime \prime}\left(M, x_{0}, u\right)$ and $I T^{\prime \prime}\left(M, x_{0}, u\right)$ are closed cones, and are convex if $M$ is convex. The cones $T^{\prime \prime}\left(M, x_{0}, u\right), A^{\prime \prime}\left(M, x_{0}, u\right)$ were proposed by Penot [35]. $T^{2}\left(M, x_{0}, u\right), A^{2}\left(M, x_{0}, u\right)$ and $I T^{2}\left(M, x_{0}, u\right)$ are closed sets, but not necessarily cones. If $M$ is convex, then $A^{2}\left(M, x_{0}, u\right)$ is convex, while $T^{2}\left(M, x_{0}, u\right)$ may not be convex.

In next proposition some known properties of second-order tangent sets are collected (see more details in $[8,13,21,24,25,35])$.

Proposition 2.4. Let $M \subseteq X$ and $x_{0}, u \in X$.

(i) $T^{2}\left(M, x_{0}, 0\right)=T^{\prime \prime}\left(M, x_{0}, 0\right)=T\left(T\left(M, x_{0}\right), 0\right)=T\left(M, x_{0}\right)$.

Let, in addition, $M$ be convex and $u \in T\left(M, x_{0}\right)$. Then, the following assertions hold

(ii) $T\left(T\left(M, x_{0}\right), u\right)=$ clcone $\left(\operatorname{cone}\left(M-x_{0}\right)-u\right)$ and $T^{2}\left(M, x_{0}, u\right) \subseteq T\left(T\left(M, x_{0}\right), u\right)$. Additionally, if $0 \in$ $T^{2}\left(M, x_{0}, u\right)$, then $T^{2}\left(M, x_{0}, u\right)=T\left(T\left(M, x_{0}\right), u\right)$.

(iii) If $T^{\prime \prime}\left(M, x_{0}, u\right) \neq \emptyset$, then $T^{\prime \prime}\left(M, x_{0}, u\right)=T\left(T\left(M, x_{0}\right), u\right)$ and $T^{2}\left(M, x_{0}, u\right) \subseteq T^{\prime \prime}\left(M, x_{0}, u\right)$

(iv) If $A^{2}\left(M, x_{0}, u\right) \neq \emptyset$, then $\operatorname{cl} I T^{2}\left(M, x_{0}, u\right)=A^{2}\left(M, x_{0}, u\right)$ and

$$
A^{2}\left(M, x_{0}, u\right)+T\left(T\left(M, x_{0}\right), u\right) \subseteq A^{2}\left(M, x_{0}, u\right) .
$$


(v) If $A^{\prime \prime}\left(M, x_{0}, u\right) \neq \emptyset$, then $\operatorname{cl} I T^{\prime \prime}\left(M, x_{0}, u\right)=A^{\prime \prime}\left(M, x_{0}, u\right)$ and

$$
A^{\prime \prime}\left(M, x_{0}, u\right)+T\left(T\left(M, x_{0}\right), u\right) \subseteq A^{\prime \prime}\left(M, x_{0}, u\right) .
$$

According to Proposition 2.4, $T^{2}\left(M, x_{0}, u\right)$ may be properly contained in $T^{\prime \prime}\left(M, x_{0}, u\right)$ and $T\left(T\left(M, x_{0}\right), u\right)$. Whenever $T^{\prime \prime}\left(M, x_{0}, u\right)$ is nonempty and $M$ is convex, $T^{\prime \prime}\left(M, x_{0}, u\right)=T\left(T\left(M, x_{0}\right), u\right)$. In general, these sets are different as shown by the following example.

Example 2.5. Let $M=\left\{\left.\left(x_{1}, x_{2}\right) \in \mathbb{R}^{2}\left|x_{2}=\right| x_{1}\right|^{\frac{5}{4}}\right\}$ and $x_{0}=(0,0)$. By directed calculations, one has $T\left(M, x_{0}\right)=\left\{\left(u_{1}, u_{2}\right) \in \mathbb{R}^{2} \mid u_{2}=0\right\}$. Take $u=(1,0), T^{2}\left(M, x_{0}, u\right)=\emptyset, T\left(T\left(M, x_{0}\right), u\right)=\mathbb{R} \times\{0\}$, and $T^{\prime \prime}\left(M, x_{0}, u\right)=\mathbb{R} \times \mathbb{R}_{+}$. Thus, we have $T^{2}\left(M, x_{0}, u\right) \subsetneq T\left(T\left(M, x_{0}\right), u\right) \subsetneq T^{\prime \prime}\left(M, x_{0}, u\right)$.

\section{Generalized SECOND-ORDER ASymptotic CONTINGEnT EPIDERIVATIVE}

Definition 3.1. Let $\Phi: X \rightrightarrows Y,\left(x_{0}, y_{0}\right) \in \operatorname{gph} \Phi$, and $(u, v) \in X \times Y$.

(i) ([18]). The contingent epiderivative of $\Phi$ at $\left(x_{0}, y_{0}\right)$ is a single-valued map $D \Phi\left(x_{0}, y_{0}\right): X \rightarrow Y$ defined by epi $D \Phi\left(x_{0}, y_{0}\right):=T\left(\operatorname{epi} \Phi,\left(x_{0}, y_{0}\right)\right)$.

(ii) ([6]). The generalized contingent epiderivative of $\Phi$ at $\left(x_{0}, y_{0}\right)$ is a set-valued map $D_{g} \Phi\left(x_{0}, y_{0}\right): X \rightrightarrows Y$ defined by $D_{g} \Phi\left(x_{0}, y_{0}\right)(x):=\operatorname{Min}_{C}\left\{y \in Y \mid(x, y) \in T\left(\operatorname{epi} \Phi,\left(x_{0}, y_{0}\right)\right)\right\}$.

(iii) ([19]). The generalized second-order contingent epiderivative of $\Phi$ at $\left(x_{0}, y_{0}\right)$ in direction $(u, v)$ is a setvalued map $D_{g}^{2} \Phi\left(x_{0}, y_{0}, u, v\right): X \rightrightarrows Y$ defined by

$$
D_{g}^{2} \Phi\left(x_{0}, y_{0}, u, v\right)(x):=\operatorname{Min}_{C}\left\{y \in Y \mid(x, y) \in T^{2}\left(\operatorname{epi} \Phi,\left(x_{0}, y_{0}\right),(u, v)\right)\right\} .
$$

(iv) ([32]). The generalized composed second-order contingent epiderivative of $\Phi$ at $\left(x_{0}, y_{0}\right)$ in direction $(u, v)$ is a set-valued map $D_{g}^{c(2)} \Phi\left(x_{0}, y_{0}, u, v\right): X \rightrightarrows Y$ defined by

$$
D_{g}^{c(2)} \Phi\left(x_{0}, y_{0}, u, v\right)(x):=\operatorname{Min}_{C}\left\{y \in Y \mid(x, y) \in T\left(T\left(\operatorname{epi} \Phi,\left(x_{0}, y_{0}\right)\right),(u, v)\right)\right\} .
$$

In general, the second-order contingent set, composed second-order contingent set and asymptotic secondorder tangent cone are different (see Example 2.5). Since the composed second-order contingent set $T\left(T\left(M, x_{0}\right), u\right)$ is the tangent cone of the tangent cone of the set $M$ at $x_{0}$ at direction $u$, then it does not contain some information concerning the directions of $M$. We propose a generalized asymptotic second-order contingent epiderivative of a set-valued map as follows.

Definition 3.2. Let $\Phi: X \rightrightarrows Y,\left(x_{0}, y_{0}\right) \in \operatorname{gph} \Phi$, and $(u, v) \in X \times Y$. The generalized asymptotic second-order contingent epiderivative of $\Phi$ at $\left(x_{0}, y_{0}\right)$ in direction $(u, v)$ is a set-valued map $D_{g}^{\prime \prime} \Phi\left(x_{0}, y_{0}, u, v\right): X \rightrightarrows Y$ defined by

$$
D_{g}^{\prime \prime} \Phi\left(x_{0}, y_{0}, u, v\right)(x):=\operatorname{Min}_{C}\left\{y \in Y \mid(x, y) \in T^{\prime \prime}\left(\operatorname{epi} \Phi,\left(x_{0}, y_{0}\right),(u, v)\right)\right\} .
$$

In the next example, the generalized second-order epiderivatives mentioned in Definitions 3.1 and 3.2 are different, and the second-order contingent epiderivative defined in Definition 3.1 does not exist.

Example 3.3. Let $C=\mathbb{R}_{+} \times\{0\}, \Phi: \mathbb{R} \rightrightarrows \mathbb{R}^{2}$ be defined by $\Phi(x):=\left\{\left.\left(y_{1}, y_{2}\right) \in \mathbb{R}^{2}\left|y_{1} \geq x^{2}, y_{2}=\right| x\right|^{\frac{3}{2}}\right\}$, $x_{0}=0$, and $y_{0}=(0,0)$. Thus, one gets $T\left(\operatorname{epi} \Phi,\left(x_{0}, y_{0}\right)\right)=\left\{(u, v) \in \mathbb{R} \times \mathbb{R}^{2} \mid u \in \mathbb{R}, v_{1} \geq 0, v_{2}=0\right\}$. Take $(u, v)=$ $(1,(0,0)), T^{2}\left(\operatorname{epi} \Phi,\left(x_{0}, y_{0}\right),(u, v)\right)=\emptyset, T\left(T\left(\operatorname{epi} \Phi,\left(x_{0}, y_{0}\right)\right),(u, v)\right)=\left\{(x, y) \in \mathbb{R} \times \mathbb{R}^{2} \mid x \in \mathbb{R}, y_{1} \geq 0, y_{2}=0\right\}$, and $T^{\prime \prime}\left(\operatorname{epi} \Phi,\left(x_{0}, y_{0}\right),(u, v)\right)=\left\{(x, y) \in \mathbb{R} \times \mathbb{R}^{2} \mid x \in \mathbb{R}, y_{1} \geq 0, y_{2} \geq 0\right\}$. From the Definitions 3.1 and 3.2 , we have $D_{g}^{2} \Phi\left(x_{0}, y_{0}, u, v\right)(x)=\emptyset, D_{g}^{c(2)} \Phi\left(x_{0}, y_{0}, u, v\right)(x)=\{(0,0)\}$, and $D_{g}^{\prime \prime} \Phi\left(x_{0}, y_{0}, u, v\right)(x)=\left\{\left(y_{1}, y_{2}\right) \in \mathbb{R}^{2} \mid\right.$ $\left.y_{1}=0, y_{2} \geq 0\right\}$. Therefore,

$$
D_{g}^{2} \Phi\left(x_{0}, y_{0}, u, v\right)(x) \subsetneq D_{g}^{c(2)} \Phi\left(x_{0}, y_{0}, u, v\right)(x) \subsetneq D_{g}^{\prime \prime} \Phi\left(x_{0}, y_{0}, u, v\right)(x) .
$$


For $\left(x_{0}, y_{0}\right) \in \operatorname{epi} \Phi$ and $(u, v) \in X \times Y$, we will use the following notations

$$
\begin{aligned}
& E^{2}(x):=\left\{y \in Y \mid(x, y) \in T^{2}\left(\operatorname{epi} \Phi,\left(x_{0}, y_{0}\right),(u, v)\right)\right\}, \\
& E^{\prime \prime}(x):=\left\{y \in Y \mid(x, y) \in T^{\prime \prime}\left(\operatorname{epi} \Phi,\left(x_{0}, y_{0}\right),(u, v)\right)\right\} .
\end{aligned}
$$

The following proposition gives relationships between the epigraph of the second-order contingent epiderivative of a set-valued mapping and the second-order tangent set of the epigraph of this mapping. The proof is similar to that of Proposition 2.4 in $[6,19,32]$ and therefore is omitted.

Proposition 3.4. Let $\Phi: X \rightrightarrows Y,\left(x_{0}, y_{0}\right) \in \operatorname{gph} \Phi$ and $(u, v) \in X \times Y$. If $D_{g}^{\prime \prime} \Phi\left(x_{0}, y_{0}, u, v\right)(x)$ exists and $E^{\prime \prime}(x)$ has the domination property for all $x \in X$, then epi $D_{g}^{\prime \prime} \Phi\left(x_{0}, y_{0}, u, v\right)=T^{\prime \prime}\left(\operatorname{epi} \Phi,\left(x_{0}, y_{0}\right),(u, v)\right)$.

The next statement presents relationships between the generalized contingent epiderivative mentioned in Definitions 3.1 and 3.2.

Proposition 3.5. Let $\Phi: X \rightrightarrows Y,\left(x_{0}, y_{0}\right) \in \operatorname{gph} \Phi,(u, v) \in X \times Y$, and $x \in X$.

(i) $D_{g}^{2} \Phi\left(x_{0}, y_{0}, 0,0\right)(x)=D_{g}^{c(2)} \Phi\left(x_{0}, y_{0}, 0,0\right)(x)=D_{g}^{\prime \prime} \Phi\left(x_{0}, y_{0}, 0,0\right)(x)=D_{g} \Phi\left(x_{0}, y_{0}\right)(x)$.

Let, in addition, $\Phi$ be $C$-convex and $T^{\prime \prime}\left(\operatorname{epi} \Phi,\left(x_{0}, y_{0}\right),(u, v)\right) \neq \emptyset$, then

(ii) $D_{g}^{c(2)} \Phi\left(x_{0}, y_{0}, u, v\right)(x)=D_{g}^{\prime \prime} \Phi\left(x_{0}, y_{0}, u, v\right)(x)$. Additionally, if $(0,0) \in T^{2}\left(\operatorname{epi} \Phi,\left(x_{0}, y_{0}\right),(u, v)\right)$, then $D_{g}^{2} \Phi\left(x_{0}, y_{0}, u, v\right)(x)=D_{g}^{c(2)} \Phi\left(x_{0}, y_{0}, u, v\right)(x)=D_{g}^{\prime \prime} \Phi\left(x_{0}, y_{0}, u, v\right)(x)$.

(iii) If the sets $E^{2}(x)$ and $E^{\prime \prime}(x)$ enjoy the domination property, then

$$
D_{g}^{2} \Phi\left(x_{0}, y_{0}, u, v\right)(x) \subseteq D_{g}^{\prime \prime} \Phi\left(x_{0}, y_{0}, u, v\right)(x)+C .
$$

Proof. (i) This follows from Proposition 2.4(i) and Definitions 3.1 and 3.2.

Next let $\Phi$ be $C$-convex. Then, epi $\Phi$ is a convex set.

(ii) By Proposition 2.4(iii), one has $T^{\prime \prime}\left(\operatorname{epi} \Phi,\left(x_{0}, y_{0}\right),(u, v)\right)=T\left(T\left(\operatorname{epi} \Phi,\left(x_{0}, y_{0}\right)\right),(u, v)\right)$. From Definition 3.1, one has $D_{g}^{\prime \prime} \Phi\left(x_{0}, y_{0}, u, v\right)(x)=D_{g}^{c(2)} \Phi\left(x_{0}, y_{0}, u, v\right)(x)$. Furthermore, if $(0,0) \in T^{2}\left(\right.$ epi $\left.\Phi,\left(x_{0}, y_{0}\right),(u, v)\right)$, by Proposition 2.4, $T^{2}\left(\operatorname{epi} \Phi,\left(x_{0}, y_{0}\right),(u, v)\right)=T\left(T\left(\operatorname{epi} \Phi,\left(x_{0}, y_{0}\right)\right),(u, v)\right)=T^{\prime \prime}\left(\operatorname{epi} \Phi,\left(x_{0}, y_{0}\right),(u, v)\right)$. Thus, we have $D_{g}^{2} \Phi\left(x_{0}, y_{0}, u, v\right)(x)=D_{g}^{c(2)} \Phi\left(x_{0}, y_{0}, u, v\right)(x)=D_{g}^{\prime \prime} \Phi\left(x_{0}, y_{0}, u, v\right)(x)$.

(iii) By Proposition 2.4(iii), $T^{2}\left(\operatorname{epi} \Phi,\left(x_{0}, y_{0}\right),(u, v) \subseteq \subseteq T^{\prime \prime}\left(\operatorname{epi} \Phi,\left(x_{0}, y_{0}\right),(u, v)\right)\right.$. Since $E^{2}(x)$ and $E^{\prime \prime}(x)$ possess the domination property, by Proposition 2.4, epi $D_{g}^{2} \Phi\left(x_{0}, y_{0}, u, v\right) \subseteq$ epi $D_{g}^{\prime \prime} \Phi\left(x_{0}, y_{0}, u, v\right)$. This implies that $D_{g}^{2} \Phi\left(x_{0}, y_{0}, u, v\right)(x) \subseteq D_{g}^{\prime \prime} \Phi\left(x_{0}, y_{0}, u, v\right)(x)+C$.

The following proposition indicates some existence criteria for generalized second-order asymptotic contingent epiderivatives, similar to those in Theorem 2 in [6] and Theorem 3.1 in [32].

Proposition 3.6. Let $\Phi: X \rightrightarrows Y,\left(x_{0}, y_{0}\right) \in \operatorname{gph} \Phi,(u, v) \in X \times Y$, and $Y$ be partially ordered by a Daniel pointed, closed and convex cone $C$. For every $x \in X$, the $C$-lower boundedness of $E^{\prime \prime}(x)$ implies the existence of $D_{g}^{\prime \prime} \Phi\left(x_{0}, y_{0}, u, v\right)(x)$.

Proof. Since the asymptotic second-order tangent cone is always a closed cone and $E^{\prime \prime}(x)$ is $C$-lower bounded for every $x \in X$, by the existence theorem for minimal elements in Luc [33], Min $E^{\prime \prime}(x)$ is nonempty, i.e., $D_{g}^{\prime \prime} \Phi\left(x_{0}, y_{0}, u, v\right)(x)$ exists.

Next, we give another existence results for generalized second-order asymptotic contingent epiderivatives by using a free disposal hypothesis. We recall (see [5,22,23]) that a closed set $M \subseteq Y$ satisfies the free disposal hypothesis with respect to a closed set $Z$ containing 0 , if $M-Z \subseteq M$. 
Lemma 3.7. Suppose that $M \subseteq Y$ satisfies the free disposal hypothesis with respect to a closed cone $Z$. Then, $Z \subseteq-I T(M, y)$ for all $y \in M$.

Proof. Let $z, z_{n} \in Z$ and $t_{n}>0$ such that $z_{n} \rightarrow z$ and $t_{n} \rightarrow 0^{+}$. As $Z$ is a cone, $t_{n} z_{n} \in Z$. By the free disposal hypothesis, one gets $y+t_{n}\left(-z_{n}\right) \in M$ for $y \in M$. This implies that $-z \in I T(M, y)$.

Proposition 3.8. Let $\Phi: X \rightrightarrows Y,\left(x_{0}, y_{0}\right) \in \operatorname{gph} \Phi,(u, v) \in X \times Y$, and $Y$ be partially ordered by a closed and convex cone $C$. Suppose that $E^{\prime \prime}(x)$ satisfies the free disposal hypothesis with respect to $-C$ and either of the following tangential relations holds at $y \in E^{\prime \prime}(x): T^{b}\left(E^{\prime \prime}(x), y\right) \cap(-C)=\{0\}$ or $\operatorname{IT}\left(E^{\prime \prime}(x), y\right)$ does not contain any line. Then, $y \in D_{g}^{\prime \prime} \Phi\left(x_{0}, y_{0}, u, v\right)(x)$.

Proof. We need to prove that $y \in \operatorname{Min}_{C} E^{\prime \prime}(x)$, meaning that $\left(E^{\prime \prime}(x)-y\right) \cap(-C)=\{0\}$. Suppose there is a nonzero $z \in\left(E^{\prime \prime}(x)-y\right) \cap(-C)$. As $E^{\prime \prime}(x)$ satisfies the free disposal hypothesis with respect to $-C$, for all $t \in[0,1], y+z-t z \in E^{\prime \prime}(x)-(-C) \subseteq E^{\prime \prime}(x)$. So, one has $y+t z \in E^{\prime \prime}(x)$ for all $t \in[0,1]$. Thus, $z \in I T\left(E^{\prime \prime}(x), y\right) \subseteq T^{b}\left(E^{\prime \prime}(x), y\right)$ and then $z \in T^{b}\left(E^{\prime \prime}(x), y\right) \cap(-C)$. If $T^{b}\left(E^{\prime \prime}(x), y\right) \cap(-C)=\{0\}$, then $z=0$, a contradiction. If this intersection properly contains 0 , by Lemma 3.7, one has $C \subseteq I T\left(E^{\prime \prime}(x), y\right)$ and hence $-z \in I T\left(E^{\prime \prime}(x), y\right)$. Because $I T\left(E^{\prime \prime}(x), y\right)$ is a cone and does not contain any line, we obtain again the contradiction $z=0$.

Next is an example to illustrate Proposition 3.8.

Example 3.9. Consider the set-valued map $\Phi: \mathbb{R} \rightrightarrows \mathbb{R}^{2}$ defined by

$$
\Phi(x)=\left\{\left(y_{1}, y_{2}\right) \in \mathbb{R}^{2} \mid y_{1}=x \sin (\sqrt{|x|}), y_{1}+y_{2} \geq x\right\}
$$

$C=\mathbb{R}_{+}^{2}$, and $x_{0}=0, y_{0}=(0,0)$. Direct computations give $T\left(\right.$ epi $\left.\Phi,\left(x_{0}, y_{0}\right)\right)=\left\{\left(u,\left(v_{1}, v_{2}\right)\right) \in \mathbb{R} \times \mathbb{R}^{2} \mid v_{1} \geq\right.$ $\left.0, v_{1}+v_{2} \geq u\right\}$. Take $\left(u,\left(v_{1}, v_{2}\right)\right)=(1,(0,1)) \in T\left(\operatorname{epi} \Phi,\left(x_{0}, y_{0}\right)\right)$, one gets

$$
T^{\prime \prime}\left(\operatorname{epi} \Phi,\left(x_{0}, y_{0}\right),(u, v)\right)=\left\{\left(x,\left(y_{1}, y_{2}\right)\right) \in \mathbb{R} \times \mathbb{R}^{2} \mid y_{1} \geq 0, y_{1}+y_{2} \geq x\right\} .
$$

Then for every $x \in \mathbb{R}, E^{\prime \prime}(x)=\left\{\left(y_{1}, y_{2}\right) \in \mathbb{R}^{2} \mid y_{1} \geq 0, y_{1}+y_{2} \geq x\right\}$. It follows from Definition 3.2 that

$$
D_{g}^{\prime \prime} \Phi\left(x_{0}, y_{0}, u, v\right)(x)=\left\{\left(y_{1}, y_{2}\right) \in \mathbb{R}^{2} \mid y_{1} \geq 0, y_{1}+y_{2}=x\right\} .
$$

Now we apply Proposition 3.8 to check the above argument. Firstly, we see that, for every $x \in \mathbb{R}, E^{\prime \prime}(x)$ satisfies the free disposal hypothesis with respect to $-C$.

Next, we consider two cases for the choice of $\left(\bar{y}_{1}, \bar{y}_{2}\right) \in\left\{\left(y_{1}, y_{2}\right) \in \mathbb{R}^{2} \mid y_{1} \geq 0, y_{1}+y_{2}=x\right\}$.

- First case: $\bar{y}_{1}>0$, by directly calculating, one has $T^{\mathrm{b}}\left(E^{\prime \prime}(x),\left(\bar{y}_{1}, \bar{y}_{2}\right)\right)=\left\{\left(w_{1}, w_{2}\right) \in \mathbb{R}^{2} \mid w_{1}+w_{2} \geq 0\right\}$, hence $T^{b}\left(E^{\prime \prime}(x),\left(\bar{y}_{1}, \bar{y}_{2}\right)\right) \cap(-C)=\{0\}$. By Proposition 3.8,

$$
\left\{\left(y_{1}, y_{2}\right) \in \mathbb{R}^{2} \mid y_{1}>0, y_{1}+y_{2}=x\right\} \subseteq D_{g}^{\prime \prime} \Phi\left(x_{0}, y_{0}, u, v\right)(x) .
$$

- Second case: $\bar{y}_{1}=0$, by Definition 2.3(i), $\operatorname{IT}\left(E^{\prime \prime}(x),\left(\bar{y}_{1}, \bar{y}_{2}\right)\right)=\left\{\left(w_{1}, w_{2}\right) \in \mathbb{R}^{2} \mid w_{1}>0, w_{1}+w_{2}>0\right\}$, and it does not contain any line. According to Proposition 3.8,

$$
\left\{\left(y_{1}, y_{2}\right) \in \mathbb{R}^{2} \mid y_{1}=0, y_{2}=x\right\} \subseteq D_{g}^{\prime \prime} \Phi\left(x_{0}, y_{0}, u, v\right)(x) .
$$

Some properties of generalized second-order asymptotic contingent epiderivatives are collected in the following.

Proposition 3.10. Let $\Phi: X \rightrightarrows Y,\left(x_{0}, y_{0}\right) \in \operatorname{gph} \Phi,(u, v) \in X \times Y$, and $x \in X$. Then, 
(i) $D_{g}^{\prime \prime} \Phi\left(x_{0}, y_{0}, u, v\right)(\cdot)$ is strictly positively homogeneous.

Moreover, if $\Phi$ is $C$-convex, and $E^{\prime \prime}(x)$ has the domination property, then

(ii) $D_{g}^{\prime \prime} \Phi\left(x_{0}, y_{0}, u, v\right)$ is subadditive;

(iii) for $v \in \Phi(u)+C, \Phi(x)-y_{0} \subseteq D_{g}^{\prime \prime} \Phi\left(x_{0}, y_{0}, u-x_{0}, v-y_{0}\right)\left(x-x_{0}\right)+C$.

Proof. The proof of (i) and (ii) are similar to that of Theorem 3.2 in [32].

(iii) For any $y \in \Phi(x)$ and sequence $\left(t_{n}, r_{n}\right) \downarrow(0,0)$ such that $t_{n} r_{n}^{-1} \downarrow 0$, since $F$ is $C$-convex, one has

$$
\begin{aligned}
y_{0}+2 t_{n}\left(v-y_{0}\right) & =2 t_{n} v+\left(1-2 t_{n}\right) y_{0} \in 2 t_{n} \Phi(u)+\left(1-2 t_{n}\right) \Phi\left(x_{0}\right)+C \subseteq \Phi\left(2 t_{n} u+\left(1-2 t_{n}\right) x_{0}\right)+C, \\
y_{0}+t_{n} r_{n}\left(y-y_{0}\right) & =t_{n} r_{n} y+\left(1-t_{n} r_{n}\right) y_{0} \in t_{n} r_{n} \Phi(x)+\left(1-t_{n} r_{n}\right) \Phi\left(x_{0}\right)+C \subseteq \Phi\left(t_{n} r_{n} x+\left(1-t_{n} r_{n}\right) x_{0}\right)+C .
\end{aligned}
$$

Therefore,

$$
\begin{aligned}
y_{0}+t_{n}\left(v-y_{0}\right)+\frac{1}{2} t_{n} r_{n}\left(y-y_{0}\right) & \in \frac{1}{2} \Phi\left(2 t_{n} u+\left(1-2 t_{n}\right) x_{0}\right)+\frac{1}{2} \Phi\left(t_{n} r_{n} x+\left(1-t_{n} r_{n}\right) x_{0}\right)+C \\
& \subseteq \Phi\left(x_{0}+t_{n}\left(u-x_{0}\right)+\frac{1}{2} t_{n} r_{n}\left(x-x_{0}\right)\right)+C .
\end{aligned}
$$

This implies that $\left(x-x_{0}, y-y_{0}\right) \in T^{\prime \prime}\left(\operatorname{epi} \Phi,\left(x_{0}, y_{0}\right),\left(u-x_{0}, v-y_{0}\right)\right)$. Moreover, as $E^{\prime \prime}\left(x-x_{0}\right)$ has the domination property, by Proposition 3.4(iii), one has epi $D_{g}^{\prime \prime} \Phi\left(x_{0}, y_{0}, u-x_{0}, v-y_{0}\right)=T^{\prime \prime}\left(\operatorname{epi} \Phi,\left(x_{0}, y_{0}\right),\left(u-x_{0}, v-y_{0}\right)\right)$, and hence $\left(x-x_{0}, y-y_{0}\right) \in \operatorname{epi} D_{g}^{\prime \prime} \Phi\left(x_{0}, y_{0}, u-x_{0}, v-y_{0}\right)$. Therefore,

$$
y-y_{0} \in D_{g}^{\prime \prime} \Phi\left(x_{0}, y_{0}, u-x_{0}, v-y_{0}\right)\left(x-x_{0}\right)+C .
$$

The proof is complete.

\section{SECOND-ORDER KKT MULTIPLIERS}

In this section, let $C \subseteq Y$ be a pointed, closed and convex cone with nonempty interior, which defines a partial order on $Y$. Let $D$ be a closed convex cone with nonempty interior in $Z$. Our set-valued vector optimization problem is

$$
\text { (P) } \quad \operatorname{Min}_{C} F(x) \text { s.t. } G(x) \cap(-D) \neq \emptyset,
$$

where $F: X \rightrightarrows Y, G: X \rightrightarrows Z$ are nonempty-valued. Let $\Omega:=\{x \in X \mid G(x) \cap(-D) \neq \emptyset\}$ denote the feasible set. We denote $D\left(z_{0}\right):=\operatorname{cone}\left(D+z_{0}\right)$ and $(F, G)(x)=F(x) \times G(x)$.

For a cone $C \subseteq Y$ (resp, $D \subseteq Z$ ), the dual cone and its quasi-interior are, resp,

$$
\begin{aligned}
C^{*} & =\left\{y^{*} \in Y^{*} \mid\left\langle y^{*}, c\right\rangle \geq 0, \forall c \in C\right\}\left(\operatorname{resp}, D^{*}\right), \\
C^{* i} & =\left\{y^{*} \in Y^{*} \mid\left\langle y^{*}, c\right\rangle>0, \forall c \in C \backslash\{0\}\right\} .
\end{aligned}
$$

Then, it is not hard to check that, for $z_{0} \in-D,\left[D\left(z_{0}\right)\right]^{*}=N\left(-D, z_{0}\right)$, the normal cone of $-D$ at $z_{0}$. Note that, if $D$ is a convex cone, then $N\left(-D, z_{0}\right)=\left\{d^{*} \in D^{*} \mid\left\langle d^{*}, z_{0}\right\rangle=0\right\}$.

The following optimality notions of set-valued vector optimization are discussed in our works.

Definition 4.1. Let $x_{0} \in \Omega$ and a pair $\left(x_{0}, y_{0}\right) \in \operatorname{gph} F$.

(i) $\left(x_{0}, y_{0}\right)$ is said to be a local minimizer of $(\mathrm{P})$ if there exists a neighborhood $U$ of $x_{0}$ such that

$$
\left(F(\Omega \cap U)-y_{0}\right) \cap(-C \backslash\{0\})=\emptyset .
$$

(ii) Supposing int $C \neq \emptyset,\left(x_{0}, y_{0}\right)$ is said to be a local weak minimizer of $(\mathrm{P})$ if there exists a neighborhood $U$ of $x_{0}$ such that

$$
\left(F(\Omega \cap U)-y_{0}\right) \cap(-\operatorname{int} C)=\emptyset .
$$


(iii) $([4]) \cdot\left(x_{0}, y_{0}\right)$ is said to be a local Benson-proper minimizer of $(\mathrm{P})$ if there exist a neighborhood $U$ of $x_{0}$ such that

$$
\operatorname{clcone}\left(F(\Omega \cap U)+C-y_{0}\right) \cap(-C)=\{0\} .
$$

(iv) ([14]). $\left(x_{0}, y_{0}\right) \in \Omega$ is said to be a local Henig-proper minimizer of (P) if there exist a neighborhood $U$ of $x_{0}$ and a convex cone $K$ such that $C \backslash\{0\} \subseteq \operatorname{int} K$ and

$$
\left(F(\Omega \cap U)-y_{0}\right) \cap(-\operatorname{int} K)=\emptyset .
$$

If $U=X$, then we have the corresponding global solutions. For $\left(x_{0}, y_{0}\right) \in \operatorname{gph} F$ and $z_{0} \in G\left(x_{0}\right) \cap(-D)$, a triple $(u, v, w) \in X \times Y \times Z$ is termed a critical direction, denoted by $\left.(u, v, w) \in \mathcal{C}\left(x_{0}, y_{0}, z_{0}\right)\right)$, if

$$
(u,(v, w)) \in T\left(\operatorname{epi}(F, G),\left(x_{0},\left(y_{0}, z_{0}\right)\right) \text { with } v \in-\operatorname{bd} C \text { and } w \in-\operatorname{cl} D\left(z_{0}\right) .\right.
$$

Now, we establish a KKT second-order necessary condition for a local weak minimizer.

Theorem 4.2. Let $\left(x_{0}, y_{0}\right) \in \operatorname{gph} F$ be a local weak minimizer of $(\mathrm{P})$ and $z_{0} \in G\left(x_{0}\right) \cap(-D)$. For $(u, v, w) \in$ $\mathcal{C}\left(x_{0}, y_{0}, z_{0}\right)$ such that $D_{g}^{\prime \prime}(F, G)\left(x_{0},\left(y_{0}, z_{0}\right), u,(v, w)\right)(X)$ is a convex set, there exist multipliers $\left(c^{*}, d^{*}\right) \in C^{*} \times$ $N\left(-D, z_{0}\right) \backslash\{(0,0)\}$ such that $\left\langle c^{*}, v\right\rangle=\left\langle d^{*}, w\right\rangle=0$ and

$$
\left\langle c^{*}, y\right\rangle+\left\langle d^{*}, z\right\rangle \geq 0
$$

for all $(y, z) \in D_{g}^{\prime \prime}(F, G)\left(x_{0},\left(y_{0}, z_{0}\right), u,(v, w)\right)(X)$. Moreover, if the KRZ qualification condition

$$
\left\{z \in Z \mid(y, z) \in D_{g}^{\prime \prime}(F, G)\left(x_{0},\left(y_{0}, z_{0}\right), u,(v, w)\right)(X)\right\}+D\left(z_{0}\right)=Z
$$

is satisfied, then $c^{*} \neq 0$.

Proof. We claim that

$$
D_{g}^{\prime \prime}(F, G)\left(x_{0},\left(y_{0}, z_{0}\right), u,(v, w)\right)(X) \bigcap\left(I T(-C, v) \times I T^{\prime \prime}\left(-D, z_{0}, w\right)\right)=\emptyset .
$$

Suppose to the contrary the existence of $x \in X$ and $(y, z) \in Y \times Z$ such that

$$
(y, z) \in D_{g}^{\prime \prime}(F, G)\left(x_{0},\left(y_{0}, z_{0}\right), u,(v, w)\right)(x) \bigcap\left(I T(-C, v) \times I T^{\prime \prime}\left(-D, z_{0}, w\right)\right) .
$$

As $(y, z) \in D_{g}^{\prime \prime}(F, G)\left(x_{0},\left(y_{0}, z_{0}\right), u,(v, w)\right)(x)$, according to Definition 3.2, one gets $(x,(y, z)) \in T^{\prime \prime}(\operatorname{epi}(F, G)$ $\left(x_{0},\left(y_{0}, z_{0}\right), u,(v, w)\right)$. It follows from the definition of asymptotic second-order tangent cone that there exist sequences $\left(t_{n}, r_{n}\right) \downarrow(0,0): t_{n} r_{n}^{-1} \downarrow 0, x_{n} \rightarrow x$, and $z_{n} \rightarrow z$ such that

$$
\begin{aligned}
& y_{0}+t_{n} v+\frac{1}{2} t_{n} r_{n} y_{n} \in F\left(x_{0}+t_{n} u+\frac{1}{2} t_{n} r_{n} x_{n}\right)+C, \\
& z_{0}+t_{n} w+\frac{1}{2} t_{n} r_{n} z_{n} \in G\left(x_{0}+t_{n} u+\frac{1}{2} t_{n} r_{n} x_{n}\right)+D .
\end{aligned}
$$

Since $z \in I T^{\prime \prime}\left(-D, z_{0}, w\right), z_{0}+t_{n} w+\frac{1}{2} t_{n} r_{n} z_{n} \in-D$, for sufficiently large $n$. Thus, one has

$$
\left(G\left(x_{0}+t_{n} u+\frac{1}{2} t_{n} r_{n} x_{n}\right)+D\right) \cap(-D) \neq \emptyset .
$$

Moreover $D$ is a convex cone, $G\left(x_{0}+t_{n} u+\frac{1}{2} t_{n} r_{n} x_{n}\right) \cap(-D) \neq \emptyset$, i.e., $x_{0}+t_{n} u+\frac{1}{2} t_{n} r_{n} x_{n} \in \Omega$. 
On the other hand, because $y \in I T(-C, v)=I T(-\operatorname{int} C, v)$ (see [20], Prop. 2.3), for sufficiently large $n$, $t_{n} v+\frac{1}{2} t_{n} r_{n} y_{n} \in-\operatorname{int} C$. Therefore,

$$
\left(F\left(x_{0}+t_{n} u+\frac{1}{2} t_{n} r_{n} x_{n}\right)-y_{0}\right) \cap(-\operatorname{int} C) \neq \emptyset,
$$

which contradicts the weak efficiency of $\left(x_{0}, y_{0}\right)$.

From the equality (4.1) and the convexity assumption, the standard separation theorem is employed to obtain $\left(c^{*}, d^{*}\right) \in Y^{*} \times Z^{*} \backslash\{(0,0)\}$ such that

$$
\left\langle c^{*}, y\right\rangle+\left\langle d^{*}, z\right\rangle \geq\left\langle c^{*}, c\right\rangle+\left\langle d^{*}, d\right\rangle
$$

for all $(y, z) \in D_{g}^{\prime \prime}(F, G)\left(x_{0},\left(y_{0}, z_{0}\right), u,(v, w)\right)(X)$ and $c \in I T(-C, v), d \in I T^{\prime \prime}\left(-D, z_{0}, w\right)$. Because $C$ is a convex cone, $I T(-C, v)=\operatorname{int}\left(\operatorname{cone}(-C-v)\right.$ ) (see [20], Prop. 2.3). It follows from (4.2) that $\left\langle c^{*}, c\right\rangle \leq 0$ for all $c \in \operatorname{cone}(-C-v)$, and so $c^{*} \in[\operatorname{cone}(C+v)]^{*}$. As $v \in-\operatorname{bd} C$, one has $c^{*} \in C^{*}$ and $\left\langle c^{*}, v\right\rangle=0$. As $\operatorname{cl} I T^{\prime \prime}\left(-D, z_{0}, w\right)=A^{\prime \prime}\left(-D, z_{0}, w\right),(4.2)$ becomes, for all $d \in A^{\prime \prime}\left(-D, z_{0}, w\right)$,

$$
\left\langle c^{*}, y\right\rangle+\left\langle d^{*}, z\right\rangle \geq\left\langle d^{*}, d\right\rangle .
$$

Since $D$ is convex, $A^{\prime \prime}\left(-D, z_{0}, w\right)=T\left(T\left(-D, z_{0}\right), w\right)$, which is a cone. Hence, $d^{*} \in\left[T\left(T\left(-D, z_{0}\right), w\right)\right]^{*}$, i.e., $d^{*} \in N\left(-D, z_{0}\right)$ and $\left\langle d^{*}, w\right\rangle=0$. Because $A^{\prime \prime}\left(-D, z_{0}, w\right)$ is a cone, we have $\left\langle c^{*}, y\right\rangle+\left\langle d^{*}, z\right\rangle \geq 0$.

The next step is to prove that $c^{*} \neq 0$ under the qualification condition. Supposing $c^{*}=0$, one gets $\left\langle d^{*}, z\right\rangle \geq 0$ for every $(y, z) \in D_{g}^{\prime \prime}(F, G)\left(x_{0},\left(y_{0}, z_{0}\right), u,(v, w)\right)(X)$. Take arbitrarily $\bar{z} \in Z$. By the qualification condition, there exist $t \geq 0, z \in\left\{z^{\prime} \in Z \mid\left(y^{\prime}, z^{\prime}\right) \in D^{\prime \prime}(F, G)\left(x_{0},\left(y_{0}, z_{0}\right), u,(v, w)\right)(X)\right\}$, and $d \in D$ such that $\bar{z}=z+t\left(d+z_{0}\right)$. Since $d^{*} \in D^{*}$ and $\left\langle d^{*}, z_{0}\right\rangle=0$, one has

$$
\left\langle d^{*}, \bar{z}\right\rangle=\left\langle d^{*}, z\right\rangle+t\left\langle d^{*}, d+z_{0}\right\rangle \geq 0 .
$$

Thus, $d^{*}=0$, a contradiction because $\left(c^{*}, d^{*}\right) \neq(0,0)$. Hence, this completes the proof.

In most known necessary conditions, $(F, G)$ and its derivatives are used, for example in $[11,13,26,27,46]$. Inspired by the idea in $[28,30]$, the Aubin property is employed to obtain a sharper second-order necessary conditions involving separately derivatives of $F$ and $G$. From there, constraint qualifications of the KurcyuszRobinson-Zowe type, not qualification condition in terms of $(F, G)$, can be invoked to get Karush-Kuhn-Tucker multiplier rules for problem $(\mathrm{P})$.

Recall that $F$ is said to be $C$-Aubin at $\left(x_{0}, y_{0}\right) \in \operatorname{gph} F$ if there exist the neighborhoods $U$ of $x_{0}, V$ of $y_{0}$, and $L>0$ such that

$$
F(x) \cap V \subseteq F\left(x^{\prime}\right)+L\left\|x-x^{\prime}\right\| \operatorname{cl} B_{Y}+C, \quad \forall x, x^{\prime} \in U .
$$

If $C=\{0\}$, this is the well-known Aubin property (known also as the pseudo-Lipschitz property or Lipschitzlike property) in [3].

Theorem 4.3. Let $\left(x_{0}, y_{0}\right) \in \operatorname{gph} F$ be a local weak minimizer of $(\mathrm{P}), z_{0} \in G\left(x_{0}\right) \cap(-D)$, and $F_{+}$be $C$-Aubin at $\left(x_{0}, y_{0}\right)$. For $\left.(u, v, w) \in \mathcal{C}\left(x_{0}, y_{0}, z_{0}\right)\right)$ such that epi $F$ is second-order asymptotic derivable at $\left(\left(x_{0}, y_{0}\right),(u, v)\right)$ and $\left(D_{g}^{\prime \prime} F\left(x_{0}, y_{0}, u, v\right), D_{g}^{\prime \prime} G\left(x_{0}, z_{0}, u, w\right)\right)(X)$ is convex, there exist multipliers $\left(c^{*}, d^{*}\right) \in C^{*} \times N\left(-D, z_{0}\right) \backslash\{(0,0)\}$ such that $\left\langle c^{*}, v\right\rangle=\left\langle d^{*}, w\right\rangle=0$ and

$$
\left\langle c^{*}, y\right\rangle+\left\langle d^{*}, z\right\rangle \geq 0
$$

for all $(y, z) \in\left(D_{g}^{\prime \prime} F\left(x_{0}, y_{0}, u, v\right), D_{g}^{\prime \prime} G\left(x_{0}, z_{0}, u, w\right)\right)(X)$. Moreover, if the KRZ constraint qualification (KRZCQ)

$$
D_{g}^{\prime \prime} G\left(x_{0}, z_{0}, u, w\right)(X)+D\left(z_{0}\right)=Z
$$

is fulfilled, then $c^{*} \neq 0$. 
Proof. We prove that, for all $x \in X$,

$$
D_{g}^{\prime \prime} F\left(x_{0}, y_{0}, u, v\right)(x) \times D_{g}^{\prime \prime} G\left(x_{0}, z_{0}, u, w\right)(x) \bigcap\left(I T(-C, v) \times I T^{\prime \prime}\left(-D, z_{0}, w\right)\right)=\emptyset .
$$

Suppose to the contrary the existence of $x \in X$ and $(y, z) \in Y \times Z$ such that $(y, z)$ lies on the left-hand side of (4.5). As $z \in D_{g}^{\prime \prime} G\left(x_{0}, z_{0}, u, w\right)(x),(x, z) \in T^{\prime \prime}\left(\operatorname{epi} G,\left(x_{0}, z_{0}\right),(u, w)\right)$. Then, there exist $\left(t_{n}, r_{n}\right) \downarrow(0,0): t_{n} r_{n}^{-1} \downarrow$ $0, x_{n} \rightarrow x$, and $z_{n} \rightarrow z$ such that $z_{0}+t_{n} w+\frac{1}{2} t_{n} r_{n} z_{n} \in G\left(x_{0}+t_{n} u+\frac{1}{2} t_{n} r_{n} x_{n}\right)+D$. As $z \in I T^{\prime \prime}\left(-D, z_{0}, w\right)$, $z_{0}+t_{n} w+\frac{1}{2} t_{n} r_{n} z_{n} \in-D$ for sufficiently large $n$. This implies that

$$
\left(G\left(x_{0}+t_{n} u+\frac{1}{2} t_{n} r_{n} x_{n}\right)+D\right) \cap(-D) \neq \emptyset .
$$

Since $D$ is a convex cone, $G\left(x_{0}+t_{n} u+\frac{1}{2} t_{n} r_{n} x_{n}\right) \cap(-D) \neq \emptyset$, i.e., $x_{0}+t_{n} u+\frac{1}{2} t_{n} r_{n} x_{n} \in \Omega$.

On the other hand, as $y \in D_{g}^{\prime \prime} F\left(x_{0}, y_{0}, u, v\right)(x),(x, y) \in T^{\prime \prime}\left(\right.$ epi $\left.F,\left(x_{0}, y_{0}\right),(u, v)\right)$. As epi $F$ is second-order asymptotic derivable at $\left(\left(x_{0}, y_{0}\right),(u, v)\right)$, one has $(x, y) \in A^{\prime \prime}\left(\right.$ epi $\left.F,\left(x_{0}, y_{0}\right),(u, v)\right)$. Hence, there exist $x_{n}^{\prime} \rightarrow x$ and $y_{n} \rightarrow y$ such that $y_{0}+t_{n} v+\frac{1}{2} t_{n} r_{n} y_{n} \in F\left(x_{0}+t_{n} u+\frac{1}{2} t_{n} r_{n} x_{n}^{\prime}\right)+C$. By employing the Aubin property of $F_{+}$at $\left(x_{0}, y_{0}\right)$, there exist a neighborhood $V$ of $y_{0}$ and $L_{F}>0$ such that, for sufficiently large $n$,

$$
F_{+}\left(x_{0}+t_{n} u+\frac{1}{2} t_{n} r_{n} x_{n}^{\prime}\right) \cap V \subseteq F\left(x_{0}+t_{n} u+\frac{1}{2} t_{n} r_{n} x_{n}\right)+\frac{1}{2} L_{F} t_{n} r_{n}\left\|x_{n}^{\prime}-x_{n}\right\| B_{Y}+C .
$$

Thus, for some $b_{n} \in B_{Y}$,

$$
y_{0}+t_{n} v+\frac{1}{2} t_{n} r_{n}\left(y_{n}-L_{F}\left\|x_{n}-x_{n}^{\prime}\right\| b_{n}\right) \in F\left(x_{0}+t_{n} u+\frac{1}{2} t_{n} r_{n} x_{n}\right)+C .
$$

As $y_{n}-L_{F}\left\|x_{n}-x_{n}^{\prime}\right\| b_{n} \rightarrow y \in I T(-C, v)$ and $I T(-C, v)=I T(-\operatorname{int} C, v)$, we have that, for large $n, t_{n} v+$ $\frac{1}{2} t_{n} r_{n}\left(y_{n}-L_{F}\left\|x_{n}-x_{n}^{\prime}\right\| b_{n}\right) \in-\operatorname{int} C$. Therefore, $\left(F\left(x_{0}+t_{n} u+\frac{1}{2} t_{n} r_{n} x_{n}\right)-y_{0}\right) \cap(-\operatorname{int} C) \neq \emptyset$. This however contradicts the weak optimality of $\left(x_{0}, y_{0}\right)$.

Argue similarly as for Theorem 4.2, by (4.5) one gets multipliers $\left(c^{*}, d^{*}\right) \in C^{*} \times N\left(-D, z_{0}\right) \backslash\{(0,0)\}$ such that $\left\langle c^{*}, v\right\rangle=\left\langle d^{*}, w\right\rangle=0$ and $\left\langle c^{*}, y\right\rangle+\left\langle d^{*}, z\right\rangle \geq 0$ for all $(y, z) \in\left(D_{g}^{\prime \prime} F\left(x_{0}, y_{0}, u, v\right), D_{g}^{\prime \prime} G\left(x_{0}, z_{0}, u, w\right)\right)(X)$. Under the (KRZCQ), similar to the proof process of Theorem 4.2 , one obtains that $c^{*} \neq 0$ and the proof is completed.

Remark 4.4. (i) In Theorem 4.3, the assumptions that $F_{+}$is $C$-Aubin at $\left(x_{0}, y_{0}\right)$ and epi $F$ is second-order asymptotic derivable at $\left(\left(x_{0}, y_{0}\right),(u, v)\right)$ can be replaced by " $G_{+}$is $D$-Aubin at $\left(x_{0}, z_{0}\right)$ and epi $G$ is secondorder asymptotic derivable at $\left(\left(x_{0}, z_{0}\right),(u, w)\right)$ ". Indeed, suppose that the relation (4.5) does not hold. Then, there are $x \in X$ and $(y, z) \in Y \times Z$ such that

$$
(y, z) \in\left(D_{g}^{\prime \prime} F\left(x_{0}, y_{0}, u, v\right)(x) \times D_{g}^{\prime \prime} G\left(x_{0}, z_{0}, u, w\right)(x)\right) \bigcap\left(I T(-C, v) \times I T^{\prime \prime}\left(-D, z_{0}, w\right)\right) .
$$

As $y \in D_{g}^{\prime \prime} F\left(x_{0}, y_{0}, u, v\right)(x)$, the are $\left(t_{n}, r_{n}\right) \downarrow(0,0): t_{n} r_{n}^{-1} \downarrow 0, x_{n} \rightarrow x$ and $y_{n} \rightarrow y$ such that

$$
y_{0}+t_{n} v+\frac{1}{2} t_{n} r_{n} y_{n} \in F\left(x_{0}+t_{n} u+\frac{1}{2} t_{n} r_{n} x_{n}\right)+C .
$$

Because $z \in D_{g}^{\prime \prime} G\left(x_{0}, z_{0}, u, w\right)(x),(x, z) \in T^{\prime \prime}\left(\operatorname{epi} G,\left(x_{0}, z_{0}\right),(u, w)\right)$. Moreover, as epi $G$ is second-order asymptotic derivable at $\left(\left(x_{0}, z_{0}\right),(u, v)\right)$, one gets $(x, z) \in A^{\prime \prime}\left(\operatorname{epi} G,\left(x_{0}, z_{0}\right),(u, w)\right)$. Then, there exist $x_{n}^{\prime} \rightarrow x$ and $z_{n} \rightarrow z$ such that $z_{0}+t_{n} w+\frac{1}{2} t_{n} r_{n} z_{n} \in G\left(x_{0}+t_{n} u+\frac{1}{2} t_{n} r_{n} x_{n}^{\prime}\right)+D$. Since $G_{+}$is $D$-Aubin at $\left(x_{0}, z_{0}\right)$, there exist a neighborhood $V$ of $z_{0}$, and $L_{G}>0$ such that, for sufficiently large $n$,

$$
G_{+}\left(x_{0}+t_{n} u+\frac{1}{2} t_{n} r_{n} x_{n}^{\prime}\right) \cap V \subseteq G\left(x_{0}+t_{n} u+\frac{1}{2} t_{n} r_{n} x_{n}\right)+\frac{1}{2} L_{G} t_{n} r_{n}\left\|x_{n}^{\prime}-x_{n}\right\| B_{Z}+D .
$$


Consequently, $z_{0}+t_{n} w+\frac{1}{2} t_{n} r_{n} z_{n} \in G\left(x_{0}+t_{n} u+\frac{1}{2} t_{n} r_{n} x_{n}\right)+\frac{1}{2} L_{G} t_{n} r_{n}\left\|x_{n}-x_{n}^{\prime}\right\| B_{Z}+D$. Thus, there exists $\bar{b}_{n} \in B_{Z}$ such that $G\left(x_{0}+t_{n} u+\frac{1}{2} t_{n} r_{n} x_{n}\right) \cap\left(z_{0}+t_{n} w+\frac{1}{2} t_{n} r_{n}\left(z_{n}-L_{G}\left\|x_{n}-x_{n}^{\prime}\right\| \bar{b}_{n}\right)-D\right) \neq \emptyset$. It follows from $z \in I T^{\prime \prime}\left(-D, z_{0}, w\right)$ and $z_{n}-L_{G}\left\|x_{n}-x_{n}^{\prime}\right\| \bar{b}_{n} \rightarrow z$ that

$$
z_{0}+t_{n} w+\frac{1}{2} t_{n} r_{n}\left(z_{n}-L_{G}\left\|x_{n}-x_{n}^{\prime}\right\| \bar{b}_{n}\right) \in-D,
$$

for sufficiently large $n$. Therefore, $G\left(x_{0}+t_{n} w+\frac{1}{2} t_{n} r_{n} x_{n}\right) \cap(-D) \neq \emptyset$, i.e., $x_{0}+t_{n} w+\frac{1}{2} t_{n} r_{n} x_{n} \in \Omega$. The rest of the proof is similar to that of Theorem 4.3.

(ii) When $F$ is $C$-convex and $G$ is $D$-convex, according to Proposition 3.5, the generalized composed contingent epiderivative coincides with the generalized asymptotic contingent epiderivative. In this case, Theorem 4.3 improves the corresponding result of Theorem 4.1 in [46], since the authors of [46] used the derivatives of a disjunction map, composed from the objective and the constraints, and the regularity assumptions involving these maps.

(iii) Note that, the authors in [28] use the cone-Aubin properties of both the objective and the constraint maps to separate the derivatives of them. In our works, we only assume this property for only the objective map or the constraint map.

(iv) For $(u,(v, w))=(0,(0,0))$, by Proposition 3.5, $D_{g}^{\prime \prime} F\left(x_{0}, y_{0}, 0,0\right)(x)=D_{g} F\left(x_{0}, y_{0}\right)(x)$ and $D_{g}^{\prime \prime} G\left(x_{0}\right.$, $\left.z_{0}, 0,0\right)(x)=D_{g} G\left(x_{0}, z_{0}\right)(x)$. Obviously, as a direct consequence of Theorem 4.3 with $(u,(v, w))=$ $(0,(0,0))$, the first-order optimality condition is immediately acquired. This condition collapses to Theorem 2.7 in [17].

Theorem 4.3 is illustrated by the following examples.

Example 4.5. Let $X=Z=\mathbb{R}^{2}, Y=\mathbb{R}, C=\mathbb{R}_{+}, D=\mathbb{R}_{+}^{2}, x_{0}=(0,0), y_{0}=(0,0), z_{0}=0$, and

$$
\begin{aligned}
& F(x)= \begin{cases}\left\{\left.y \in \mathbb{R}\left|y \geq x_{1}^{2}+\right| x_{2}\right|^{\frac{5}{4}}\right\} & \text { if } x_{1} x_{2} \geq 0, \\
\emptyset & \text { if } x_{1} x_{2}<0,\end{cases} \\
& G(x)= \begin{cases}\left\{\left(x_{1}^{2}, x_{2}^{2}\right),\left(-\left|x_{1}\right|,-\left|x_{2}\right|\right)\right\} & \text { if } x_{1} x_{2} \geq 0, \\
\emptyset & \text { if } x_{1} x_{2}<0 .\end{cases}
\end{aligned}
$$

Then, $F$ is $C$-Aubin at $\left(x_{0}, y_{0}\right)$. Direct calculations give get $T\left(\right.$ epi $\left.F,\left(x_{0}, y_{0}\right)\right)=\left\{(u, v) \in \mathbb{R}^{2} \times \mathbb{R} \mid v \geq 0\right\}$ and $T\left(\operatorname{epi} G,\left(x_{0}, z_{0}\right)\right)=\left\{(u, w) \in \mathbb{R}^{2} \times \mathbb{R}^{2}\left|w_{1} \geq-\right| u_{1}\left|, w_{2} \geq-\right| u_{2} \mid\right\}$. Take $(u, v)=((1,1), 0) \in T\left(\operatorname{epi} F,\left(x_{0}, y_{0}\right)\right)$ and $(u, w)=((1,1),(-1,-1)) \in T\left(\operatorname{epi} G,\left(x_{0}, z_{0}\right)\right)$, one has $v \in-\operatorname{bd} C, w \in-\operatorname{cl} D\left(z_{0}\right)$, hence $(u, v, w) \in$ $\left.\mathcal{C}\left(x_{0}, y_{0}, z_{0}\right)\right)$. By Definition 2.3, we get

$$
\begin{aligned}
A^{\prime \prime}\left(\operatorname{epi} F,\left(x_{0}, y_{0}\right),(u, v)\right) & =T^{\prime \prime}\left(\operatorname{epi} F,\left(x_{0}, y_{0}\right),(u, v)\right)=\left\{(x, y) \in \mathbb{R}^{2} \times \mathbb{R} \mid y \geq 0\right\}, \\
T^{\prime \prime}\left(\operatorname{epi} G,\left(x_{0}, y_{0}\right),(u, w)\right) & =\left\{(x, z) \in \mathbb{R}^{2} \times \mathbb{R}^{2} \mid z_{1} \geq-x_{1}, z_{2} \geq-x_{2}\right\} .
\end{aligned}
$$

Thus, epi $F$ is second-order asymptotic derivable at $\left(\left(x_{0}, y_{0}\right),(u, v)\right)$ and

$$
D_{g}^{\prime \prime} F\left(x_{0}, y_{0}, u, v\right)(x)=\{0\}, \quad D_{g}^{\prime \prime} G\left(x_{0}, z_{0}, u, w\right)(x)=\left\{\left(-x_{1},-x_{2}\right)\right\} .
$$

As $D_{g}^{\prime \prime} G\left(x_{0}, z_{0}, u, w\right)(X)+D\left(z_{0}\right)=\mathbb{R}^{2}$, the (KRZCQ) is satisfied.

We can verify that $\left(x_{0}, y_{0}\right)=((0,0), 0)$ is a local weak minimizer of $(\mathrm{P})$ and all the assumptions of Theorem 4.3 hold. By taking $c^{*}=1$ and $d^{*}=(0,0) \in D^{*}$, one has $\left\langle c^{*}, v\right\rangle=\left\langle d^{*}, w\right\rangle=0$ and

$$
\left\langle c^{*}, y\right\rangle+\left\langle d^{*}, z\right\rangle \geq 0
$$

for all $(y, z) \in\left(D_{g}^{\prime \prime} F\left(x_{0}, y_{0}, u, v\right), D_{g}^{\prime \prime} G\left(x_{0}, z_{0}, u, w\right)\right)(X)$. Therefore, the conclusions of Theorem 4.3 hold.

We can check that the second-order contingent derivative and epiderivative of $F$ at $\left(x_{0}, y_{0}\right)$ does not exist, then Theorem 3.1 in [19] and Theorems 3.1 and 3.2 in [28] cannot be employed. Furthermore, $F$ is not $C$-convex, hence Theorem 4.1 in [46] cannot also be used. 
In the next example, Theorem 4.3 rejects a candidate for a local weak minimizer of problem $(\mathrm{P})$.

Example 4.6. Let $X=\mathbb{R}, Y=\mathbb{R}^{2}, Z=\mathbb{R}, C=\mathbb{R}_{+}^{2}, D=\mathbb{R}_{+}$, and

$$
F(x):=\left\{\left(y_{1}, y_{2}\right) \in \mathbb{R}^{2}\left|y_{1}+y_{2} \geq-\right| x \mid\right\}, G(x):=\left\{z \in \mathbb{R} \mid z \geq x^{3}-2 x\right\} .
$$

Consider $x_{0}=0, y_{0}=(0,0)$, and $z_{0}=0$. Then, $F$ is $C$-Aubin at $\left(x_{0}, y_{0}\right)$. By direct calculations, we have

$$
T\left(\operatorname{epi} F,\left(x_{0}, y_{0}\right)\right)=\left\{(u, v) \in \mathbb{R} \times \mathbb{R}^{2}\left|v_{1}+v_{2} \geq-\right| u \mid\right\}, T\left(\operatorname{epi} G,\left(x_{0}, z_{0}\right)\right)=\{(u, w) \in \mathbb{R} \times \mathbb{R} \mid w \geq-2 u\} .
$$

Take $(u, v)=(1,(0,-1)) \in T\left(\operatorname{epi} F,\left(x_{0}, y_{0}\right)\right)$ and $(u, w)=(1,-2) \in T\left(\operatorname{epi} G,\left(x_{0}, z_{0}\right)\right)$, one gets $v \in-$ bd $C$, $w \in-\operatorname{cl} D\left(z_{0}\right)$, hence $\left.(u, v, w) \in \mathcal{C}\left(x_{0}, y_{0}, z_{0}\right)\right)$. Direct calculations yield

$$
\begin{aligned}
A^{\prime \prime}\left(\operatorname{epi} F,\left(x_{0}, y_{0}\right),(u, v)\right) & =T^{\prime \prime}\left(\operatorname{epi} F,\left(x_{0}, y_{0}\right),(u, v)\right)=\left\{(x, y) \in \mathbb{R} \times \mathbb{R}^{2} \mid y_{1}+y_{2} \geq-x\right\}, \\
T^{\prime \prime}\left(\operatorname{epi} G,\left(x_{0}, y_{0}\right),(u, w)\right) & =\{(x, z) \in \mathbb{R} \times \mathbb{R} \mid z \geq-2 x\},
\end{aligned}
$$

Consequently, epi $F$ is second-order asymptotic derivable at $\left(\left(x_{0}, y_{0}\right),(u, v)\right)$ and

$$
D_{g}^{\prime \prime} F\left(x_{0}, y_{0}, u, v\right)(x)=\left\{\left(y_{1}, y_{2}\right) \in \mathbb{R}^{2} \mid y_{1}+y_{2}=-x\right\}, \quad D_{g}^{\prime \prime} G\left(x_{0}, z_{0}, u, w\right)(x)=\{z \in \mathbb{R} \mid z=-2 x\} .
$$

Then, $\left(D_{g}^{\prime \prime} F\left(x_{0}, y_{0}, u, v\right), D_{g}^{\prime \prime} G\left(x_{0}, z_{0}, u, w\right)\right)(X)$ is a convex set and (KRZCQ) is fulfilled.

Take $x=1 \in X, y=(-1,0) \in D_{g}^{\prime \prime} F\left(x_{0}, y_{0}, u, v\right)(x)$, and $z=-2 \in D_{g}^{\prime \prime} G\left(x_{0}, z_{0}, u, w\right)(x)$. To check the necessary condition given in this theorem, we discuss all $c^{*}=\left(c_{1}, c_{2}\right) \in \mathbb{R}_{+}^{2} \backslash\{(0,0)\}$ and $d^{*} \in N\left(-D, z_{0}\right)$ with $\left\langle c^{*}, v\right\rangle=0$ and $\left\langle d^{*}, w\right\rangle=0$. One has $c_{1}>0, c_{2}=0$, and $d^{*}=0$. Then, for any $c^{*}=\left(c_{1}, 0\right) \in \mathbb{R}_{+}^{2}$ with $c_{1}>0$ and $d^{*}=0$,

$$
\left\langle c^{*}, y\right\rangle+\left\langle d^{*}, z\right\rangle=-c_{1}<0 .
$$

Theorem 4.3 ensures that $\left(x_{0}, y_{0}\right)$ is not a local weak minimizer of problem $(\mathrm{P})$.

Our constraint qualifications in Theorems 4.2 and 4.3 are form of Kurcyusz-Robinson-Zowe condition, see more details in $[12,28,30,46]$. With the help of Robinson-Ursescu open mapping theorem, a sufficient condition for the qualification (4.4) is studied. This qualification is also compared with the following constraint qualifications.

$(\mathrm{MFCQ})_{1}$ (Mangasarian-Fromovitz constraint qualification). There is $\bar{x} \in X$ such that

$$
D_{g}^{\prime \prime}\left(x_{0}, z_{0}, u, w\right)(\bar{x}) \cap(-\operatorname{int} D) \neq \emptyset .
$$

$(\mathrm{MFCQ})_{2}$ (Relaxed Mangasarian-Fromovitz constraint qualification). There is $\bar{x} \in X$ such that

$$
D_{g}^{\prime \prime}\left(x_{0}, z_{0}, u, w\right)(\bar{x}) \cap I T\left(-D, z_{0}\right) \neq \emptyset .
$$

Proposition 4.7. (i) If the graph of $D_{g}^{\prime \prime}\left(x_{0}, z_{0}, u, w\right)$ is closed and convex; $0 \in \operatorname{core} D_{g}^{\prime \prime}\left(x_{0}, z_{0}, u, w\right)(X)(\operatorname{core}(\cdot)$ stands for the algebraic interior of a set $(\cdot))$, then the (KRZCQ) is satisfied.

(ii) $(\mathrm{MFCQ})_{1} \Rightarrow(\mathrm{MFCQ})_{2} \Rightarrow(\mathrm{KRZCQ})$.

Proof. (i) By assumption, the graph of the map $\Phi$ defined by $\Phi(x):=D_{g}^{\prime \prime} G\left(x_{0}, z_{0}, u, w\right)(x)+D\left(z_{0}\right)$ is closed and convex, $0 \in \operatorname{core} \Phi(X)$, and $\Phi(X)$ is a convex set. By the Robinson-Ursescu open mapping theorem (see $[10,36,43])$, for $\bar{x} \in X$ with $0 \in \Phi(\bar{x})$, there exists $\epsilon>0$ such that $\epsilon B(0,1) \subset \Phi(\bar{x}+B(0,1))$. Therefore, $\epsilon B(0,1) \subset D_{g}^{\prime \prime} G\left(x_{0}, z_{0}, u, w\right)(X)+D\left(z_{0}\right)$. As $D_{g}^{\prime \prime} G\left(x_{0}, z_{0}, u, w\right)$ is strictly positively homogeneous and $D\left(z_{0}\right)$ is a cone, one gets (4.4). 
(ii) It is easy to verify that $-\operatorname{int} D \subseteq I T\left(-D, z_{0}\right)$ and then $(\mathrm{CQ})_{1} \Rightarrow(\mathrm{CQ})_{2}$. Next, we claim that $(\mathrm{CQ})_{2} \Rightarrow(4.4)$. By $(\mathrm{CQ})_{2}$, for $\bar{z} \in D_{g}^{\prime \prime} G\left(x_{0}, z_{0}, u, w\right)(\bar{x}) \cap I T\left(-D, z_{0}\right), z \in Z$, and large $n \in \mathbb{N}$, one has $-z_{0}-\frac{1}{n}\left(\bar{z}-\frac{1}{n} z\right) \in$ $D$. By the strictly positively homogeneous property of $D_{g}^{\prime \prime} G\left(x_{0}, z_{0}, u, w\right), n \bar{z} \in D_{g}^{\prime \prime} G\left(x_{0}, z_{0}, u, w\right)(n \bar{x})$. Thus

$$
z=n \bar{z}+n^{2}\left(-z_{0}-\frac{1}{n} \bar{z}+\frac{1}{n^{2}} z+z_{0}\right) \in D_{g}^{\prime \prime} G\left(x_{0}, z_{0}, u, w\right)(X)+D\left(z_{0}\right) .
$$

Because $z \in Z$ is arbitrary, the conditions (4) is fulfilled.

We explain Proposition 4.7(ii) by the following examples.

Example 4.8. (a) Let $C, D, G$, and $\left(x_{0}, z_{0}\right)$ be as in Example 4.6. By choosing $\bar{x}=1$, one has $D_{g}^{\prime \prime}\left(x_{0}, z_{0}, u, w\right)(\bar{x})=\{-2\} \in-$ int $D$, hence (MFCQ) $)_{1}$ holds. By Proposition 4.7(ii), (KRZCQ) also holds. Indeed, by directed calculations, we have $D_{g}^{\prime \prime} G\left(x_{0}, z_{0}, u, w\right)(\mathbb{R})+D\left(z_{0}\right)=\mathbb{R}$.

(b) (the converse of Prop. 4.7(ii) maybe not true). Let $C=\mathbb{R}_{+} \times \mathbb{R}, D=\left\{\left(z_{1}, z_{2}\right) \in \mathbb{R} \mid z_{2}=0\right\}$, and $G: \mathbb{R} \rightrightarrows \mathbb{R}^{2}$ be defined by $G(x):=\left\{\left(z_{1}, z_{2}\right) \in \mathbb{R}^{2} \mid z_{1} \geq x^{2}\right\}, x_{0}=0$, and $z_{0}=(0,0)$. Direct computations yield $T\left(\operatorname{epi} G,\left(x_{0}, z_{0}\right)\right)=\left\{(u, v) \in \mathbb{R} \times \mathbb{R}^{2} \mid u \in \mathbb{R}, v_{1} \geq 0\right\}$. For $(u, v)=(1,(0,0))$, calculations yield $T^{\prime \prime}\left(\operatorname{epi} G,\left(x_{0}, z_{0}\right),(u, v)\right)=\left\{(x, z) \in \mathbb{R} \times \mathbb{R}^{2} \mid x \in \mathbb{R}, z_{1} \geq 0\right\}$, hence $D_{g}^{\prime \prime} G\left(x_{0}, z_{0}, u, v\right)(x)=\left\{\left(z_{1}, z_{2}\right) \in\right.$ $\left.\mathbb{R}^{2} \mid z_{1}=0\right\}$. One can verify that $D_{g}^{\prime \prime} G\left(x_{0}, z_{0}, u, w\right)(\mathbb{R})+D\left(z_{0}\right)=\mathbb{R}^{2}$ and (KRZCQ) is fulfilled. However, neither $(\mathrm{MFCQ})_{1}$ nor $(\mathrm{MFCQ})_{2}$ is satisfied because int $D=I T\left(-D, z_{0}\right)=\emptyset$.

In [19], Jahn et al. proposed a second-order generalized contingent epiderivative and applied it to establish second-order necessary conditions in a primal form for a set-valued optimization problem with an abstract feasible set. In this paper, we present a KKT second-order rule for local weak minimizers using this secondorder generalized contingent epiderivative.

Theorem 4.9. Let $\left(x_{0}, y_{0}\right) \in \operatorname{gph} F$ be a local weak minimizer of $(\mathrm{P}), z_{0} \in G\left(x_{0}\right) \cap(-D)$, and $F$ be $C$ Aubin at $\left(x_{0}, y_{0}\right)$. For $\left.(u, v, w) \in \mathcal{C}\left(x_{0}, y_{0}, z_{0}\right)\right)$ such that epi $F$ is second-order derivable at $\left(\left(x_{0}, y_{0}\right),(u, v)\right)$ and $\left(D_{g}^{2} F\left(x_{0}, y_{0}, u, v\right), D_{g}^{2} G_{+}\left(x_{0}, z_{0}, u, w\right)\right)(X)$ is convex, there exist $\left(c^{*}, d^{*}\right) \in C^{*} \times N\left(-D, z_{0}\right) \backslash\{(0,0)\}$ such that $\left\langle c^{*}, v\right\rangle=\left\langle d^{*}, w\right\rangle=0$ and

$$
\left\langle c^{*}, y\right\rangle+\left\langle d^{*}, z\right\rangle \geq \sup _{d \in A^{2}\left(-D, z_{0}, w\right)}\left\langle d^{*}, d\right\rangle
$$

for all $(y, z) \in\left(D_{g}^{2} F\left(x_{0}, y_{0}, u, v\right), D_{g}^{2} G\left(x_{0}, z_{0}, u, w\right)\right)(X)$. Moreover, if the KRZ constraint qualification

$$
D_{g}^{2} G\left(x_{0}, z_{0}, u, w\right)(X)-A^{2}\left(-D, z_{0}, w\right)+D\left(z_{0}\right)=Z
$$

is fulfilled, then $c^{*} \neq 0$.

Proof. Similar to the first part of the proof of Theorem 4.3, we also get, for all $x \in X$,

$$
D_{g}^{2} F\left(x_{0}, y_{0}, u, v\right)(x) \times D_{g}^{2} G\left(x_{0}, z_{0}, u, w\right)(x) \bigcap\left(I T(-C, v) \times I T^{2}\left(-D, z_{0}, w\right)\right)=\emptyset .
$$

Hence, by the convexity assumption, the standard separation theorem gives $\left(c^{*}, d^{*}\right) \in Y^{*} \times Z^{*} \backslash\{(0,0,0)\}$ such that

$$
\left\langle c^{*}, y\right\rangle+\left\langle d^{*}, z\right\rangle \geq\left\langle c^{*}, c\right\rangle+\left\langle d^{*}, d\right\rangle
$$

for all $(y, z) \in\left(D_{g}^{2} F\left(x_{0}, y_{0}, u, v\right), D_{g}^{2} G_{+}\left(x_{0}, z_{0}, u, w\right)\right)(X)$ and $c \in I T(-C, v), d \in I T^{2}\left(-D, z_{0}, w\right)$. Since $I T(-C, v)$ is a cone, $(4.7)$ yields that $c^{*} \in C^{*}$ and $\left\langle c^{*}, v\right\rangle=0$. According to Proposition 2.4, $\operatorname{cl} I T^{2}\left(-D, z_{0}, w\right)=$ $A^{2}\left(-D, z_{0}, w\right)$ and $A^{2}\left(-D, z_{0}, w\right)+T\left(T\left(-D, z_{0}\right), w\right) \subseteq A^{2}\left(-D, z_{0}, w\right)$. Then, taking $c=0$ in (4.7) one has, for all $d \in A^{2}\left(-D, z_{0}, w\right)$ and $d^{\prime} \in T\left(T\left(-D, z_{0}\right), w\right)$,

$$
\left\langle c^{*}, y\right\rangle+\left\langle d^{*}, z\right\rangle \geq\left\langle d^{*}, d\right\rangle+\left\langle d^{*}, d^{\prime}\right\rangle
$$


Because $T\left(T\left(-D, z_{0}\right), w\right)$ is a cone, $d^{*} \in-\left[T\left(T\left(-D, z_{0}\right), w\right)\right]^{*}=\left\{d^{*} \in N\left(-D, z_{0}\right) \mid\left\langle d^{*}, w\right\rangle=0\right\}$. By letting $d^{\prime}=0$, the following inequality is acquired

$$
\left\langle c^{*}, y\right\rangle+\left\langle d^{*}, z\right\rangle \geq \sup _{d \in A^{2}\left(-D, z_{0}, w\right)}\left\langle d^{*}, d\right\rangle .
$$

Next, we verify that $c^{*} \neq 0$ under the constraint qualification. Supposing $c^{*}=0$, one obtains

$$
\left\langle d^{*}, z\right\rangle \geq \sup _{d \in A^{2}\left(-D, z_{0}, w\right)}\left\langle d^{*}, d\right\rangle
$$

for every $z \in D_{g}^{2} G_{+}\left(x_{0}, z_{0}, u, w\right)(X)$. Take arbitrarily $\bar{z} \in Z$. By the constraint qualification, there are $t \geq 0$, $z \in D_{g}^{2} G_{+}\left(x_{0}, z_{0}, u, w\right)(X), \bar{d} \in A^{2}\left(-D, z_{0}, w\right)$, and $d \in D$ such that $\bar{z}=z-\bar{d}+t\left(d+z_{0}\right)$. AS $d^{*} \in D^{*}$ and $\left\langle d^{*}, z_{0}\right\rangle=0$, one has

$$
\left\langle d^{*}, \bar{z}\right\rangle=\left\langle d^{*}, z-\bar{d}\right\rangle+t_{2}\left\langle d^{*}, d+z_{0}\right\rangle \geq \sup _{d \in A^{2}\left(-D, z_{0}, w\right)}\left\langle d^{*}, d\right\rangle-\left\langle d^{*}, \bar{d}\right\rangle \geq 0 .
$$

By the arbitrariness of $\bar{z} \in Z$, the results is a contradiction that $d^{*}=0$.

Remark 4.10. (i) Since $A^{2}\left(-D, z_{0}, w\right) \subseteq \operatorname{cl}\left[\operatorname{cone}\left[\operatorname{cone}\left(-D-z_{0}\right)-w\right]\right]$ and

$$
d^{*} \in-\left[T\left(T\left(-D, z_{0}\right), w\right)\right]^{*}=-\left[\operatorname{cl}\left[\operatorname{cone}\left[\operatorname{cone}\left(-D-z_{0}\right)-w\right]\right]\right]^{*},
$$

we have

$$
\sup _{d \in A^{2}\left(-D, z_{0}, w\right)}\left\langle d^{*}, d\right\rangle \leqslant 0 .
$$

It can be strictly negative, i.e., the envelope-like effect occurs. Of course, this supremum vanishes if $0 \in$ $A^{2}\left(-D, z_{0}, w\right)$. So, for direction $w$ satisfying this, the multiplier rule in Theorem 4.9 takes the classical form. For example, if $w \in-D\left(z_{0}\right)$, then $0 \in A^{2}\left(-D, z_{0}, w\right)$. However, Theorem 4.9 also considers critical directions $w \in-\operatorname{cl} D\left(z_{0}\right)$. For $w \in-\left(\operatorname{cl} D\left(z_{0}\right) \backslash D\left(z_{0}\right)\right)$, the envelope-like effect can occur. Since $A^{\prime \prime}\left(-D, z_{0}, w\right)$ is a cone, $0 \in A^{\prime \prime}\left(-D, z_{0}, w\right)$ and hence in Theorems 4.2 and 4.3 , this phenomenon does not occur.

(ii) If $w \in-D\left(z_{0}\right), 0 \in A^{2}\left(-D, z_{0}, w\right)$ and (4.6) becomes $D_{g}^{2} G\left(x_{0}, z_{0}, u, w\right)(X)+D\left(z_{0}\right)=Z$. Hence, the constraint qualifications (4.4) and (4.6) are of the same type, but in terms of different kinds of derivatives.

Later, we move on to sufficient conditions for some global minimizers of problem $(\mathrm{P})$.

Theorem 4.11. For $(\mathrm{P})$, let $x_{0} \in \Omega, y_{0} \in F\left(x_{0}\right), z_{0} \in G\left(x_{0}\right) \cap(-D)$, and $(v, w) \in(F, G)(u)+C \times D$. Assume that $(F, G)$ is $C \times D$-convex and $E_{(F, G)}^{\prime \prime}\left(x-x_{0}\right)$ fulfills the domination property for all $x \in \Omega$. If there exist $c^{*} \in C^{*} \backslash\{0\}$ and $d^{*} \in D^{*}$ such that $\left\langle d^{*}, z_{0}\right\rangle=0$ and

$$
\left\langle q^{*}, y\right\rangle+\left\langle d^{*}, z\right\rangle \geq 0
$$

for all $x \in \Omega$ and $(y, z) \in D_{g}^{\prime \prime}(F, G)\left(x_{0},\left(y_{0}, z_{0}\right), u-x_{0},\left(v-y_{0}, w-z_{0}\right)\right)\left(x-x_{0}\right)$, then $\left(x_{0}, y_{0}\right)$ is a global weak minimizer of $(\mathrm{P})$.

Proof. Suppose that $\left(x_{0}, y_{0}\right)$ is not a global weak minimizer of $(\mathrm{P})$, then there are $\bar{x} \in \Omega, \bar{y} \in F(\bar{x})$, and $\bar{z} \in Z$ such that

$$
\bar{y}-y_{0} \in-\operatorname{int} C \text { and } \bar{z} \in G(\bar{x}) \cap(-D) .
$$

It follows from Proposition 3.10 that, for $\bar{x} \in \Omega$,

$$
(F, G)(\bar{x})-\left(y_{0}, z_{0}\right) \subseteq D_{g}^{\prime \prime}(F, G)\left(x_{0},\left(y_{0}, z_{0}\right), u-x_{0},\left(v-y_{0}, w-z_{0}\right)\right)\left(\bar{x}-x_{0}\right)+C \times D .
$$

Thus, there exist $\left(y^{\prime}, z^{\prime}\right) \in D_{g}^{\prime \prime}(F, G)\left(x_{0},\left(y_{0}, z_{0}\right), u-x_{0},\left(v-y_{0}, w-z_{0}\right)\right)\left(\bar{x}-x_{0}\right)$ and $(\bar{c}, \bar{d}) \in C \times D$ such that $\bar{y}-y_{0}=y^{\prime}+c$ and $\bar{z}-z_{0}=z^{\prime}+d$. For $c^{*} \in C^{*} \backslash\{0\}$ and $d^{*} \in D^{*}$ with $\left\langle d^{*}, z_{0}\right\rangle=0$, one has

$$
\left\langle c^{*}, y^{\prime}\right\rangle+\left\langle d^{*}, z^{\prime}\right\rangle=\left\langle c^{*}, \bar{y}-y_{0}-c\right\rangle+\left\langle d^{*}, \bar{z}-z_{0}-d\right\rangle .
$$


Because $\bar{y}-y_{0}-c \in-\operatorname{int} C-C \subseteq-\operatorname{int} C, \bar{z}-d \in-D-D \subseteq-D$, and $\left\langle d^{*}, z_{0}\right\rangle=0$, we have $\left\langle c^{*}, \bar{y}-y_{0}-c\right\rangle<0$ and $\left\langle d^{*}, \bar{z}-z_{0}-d\right\rangle \leq 0$. Therefore,

$$
\left\langle c^{*}, y^{\prime}\right\rangle+\left\langle d^{*}, z^{\prime}\right\rangle<0
$$

which is a contradiction. Consequently, $\left(x_{0}, y_{0}\right)$ is a global weak minimizer of $(\mathrm{P})$.

Theorem 4.12. For $(\mathrm{P})$, let $x_{0} \in \Omega, y_{0} \in F\left(x_{0}\right), z_{0} \in G\left(x_{0}\right) \cap(-D)$, and $(v, w) \in(F, G)(u)+C \times D$. Assume that $(F, G)$ is $C \times D$-convex and $E_{(F, G)}^{\prime \prime}\left(x-x_{0}\right)$ fulfills the domination property for all $x \in \Omega$. If there exist $c^{*} \in C^{* i}$ and $d^{*} \in D^{*}$ such that $\left\langle d^{*}, z_{0}\right\rangle=0$ and

$$
\left\langle q^{*}, y\right\rangle+\left\langle d^{*}, z\right\rangle \geq 0
$$

for all $x \in \Omega$ and $(y, z) \in D_{g}^{\prime \prime}(F, G)\left(x_{0},\left(y_{0}, z_{0}\right), u-x_{0},\left(v-y_{0}, w-z_{0}\right)\right)\left(x-x_{0}\right)$, then $\left(x_{0}, y_{0}\right)$ is both global Henig-proper and Benson-proper minimizer of $(\mathrm{P})$.

Proof. (i) Henig-proper minimizer. According to Proposition 3.10, for all $x \in \Omega$,

$$
(F, G)(x)-\left(y_{0}, z_{0}\right) \subseteq D_{g}^{\prime \prime}(F, G)\left(x_{0},\left(y_{0}, z_{0}\right), u-x_{0},\left(v-y_{0}, w-z_{0}\right)\right)\left(x-x_{0}\right)+C \times D .
$$

For $x \in \Omega$ and $(y, z) \in(F, G)(x)$, there exist $\left(y^{\prime}, z^{\prime}\right) \in D_{g}^{\prime \prime}(F, G)\left(x_{0},\left(y_{0}, z_{0}\right), u-x_{0},\left(v-y_{0}, w-z_{0}\right)\right)\left(x-x_{0}\right)$, and $(c, d) \in C \times D$ such that $y-y_{0}=y^{\prime}+q$ and $z-z_{0}=z^{\prime}+d$. Given $c^{*} \in C^{* i}$ and $d^{*} \in D^{*}$ satisfying the assumptions of the theorem, one gets

$$
\begin{aligned}
\left\langle c^{*}, y\right\rangle+\left\langle d^{*}, z\right\rangle & =\left\langle c^{*}, y_{0}\right\rangle+\left\langle d^{*}, z_{0}\right\rangle+\left\langle c^{*}, y^{\prime}\right\rangle+\left\langle d^{*}, z^{\prime}\right\rangle+\left\langle c^{*}, c\right\rangle+\left\langle d^{*}, d\right\rangle, \\
\left\langle c^{*}, y-y_{0}\right\rangle+\left\langle d^{*}, z\right\rangle & =\left\langle d^{*}, z_{0}\right\rangle+\left\langle c^{*}, y^{\prime}\right\rangle+\left\langle d^{*}, z^{\prime}\right\rangle+\left\langle c^{*}, c\right\rangle+\left\langle d^{*}, d\right\rangle .
\end{aligned}
$$

By assumption, one has $\left\langle d^{*}, z_{0}\right\rangle=0$ and $\left\langle c^{*}, y^{\prime}\right\rangle+\left\langle d^{*}, z^{\prime}\right\rangle+\left\langle c^{*}, c\right\rangle+\left\langle d^{*}, d\right\rangle \geq 0$. So, for all $x \in \Omega$ and $(y, z) \in(F, G)(x)$, one gets

$$
\left\langle c^{*}, y-y_{0}\right\rangle+\left\langle d^{*}, z\right\rangle \geq 0 .
$$

Assume that $x_{0}$ is not a global Henig-proper solution, i.e., for any pointed convex cone $H$ with $C \backslash\{0\} \subseteq$ $\operatorname{int} H$, one can find $x_{H} \in \Omega$ such that $\left(F\left(x_{H}\right)-y_{0}\right) \cap(-H \backslash\{0\}) \neq \emptyset$. Setting a set $\mathcal{H}:=\{y \in Y \mid$ $\left.\left\langle c^{*}, y-y_{0}\right\rangle>0\right\} \cup\{0\}$, we see that $\mathcal{H}$ is a convex cone with $C \backslash\{0\} \subseteq \operatorname{int} \mathcal{H}$. Thus, one has $x_{\mathcal{H}} \in \Omega$ satisfying $\left(F\left(x_{\mathcal{H}}\right)-y_{0}\right) \cap(-\mathcal{H} \backslash\{0\}) \neq \emptyset$. Consequently, there exist $y^{\prime}-y_{0} \in\left(F\left(x_{\mathcal{H}}\right)-y_{0}\right) \cap(-\mathcal{H} \backslash\{0\})$ and $z^{\prime} \in G\left(x_{\mathcal{H}}\right) \cap(-D)$ such that

$$
\left\langle c^{*}, y^{\prime}-y_{0}\right\rangle+\left\langle d^{*}, z^{\prime}\right\rangle<0,
$$

a contradiction.

(ii) Benson-proper minimizer. Similar to (i), one has $\left\langle c^{*}, y-y_{0}\right\rangle+\left\langle d^{*}, z\right\rangle \geq 0$ for all $x \in \Omega$ and $(y, z) \in(F, G)(x)$. If $x_{0}$ is not a global Benson-proper solution, then there exists

$$
y \in(-C \backslash\{0\}) \cap \operatorname{clcone}\left(F(\Omega)-y_{0}+C\right) .
$$

Therefore, there exist $x_{n} \in \Omega, y_{n} \in F\left(x_{n}\right), z_{n} \in G\left(x_{n}\right) \cap(-D), c_{n} \in C$, and $t_{n}>0$ such that $\lim _{n \rightarrow \infty} t_{n}\left(y_{n}-\right.$ $\left.y_{0}+c_{n}\right)=y$. Hence, $\lim _{n \rightarrow \infty}\left\langle c^{*}, t_{n}\left(y_{n}-y_{0}+c_{n}\right)\right\rangle=\left\langle c^{*}, y\right\rangle$. As $c^{*} \in C^{* i}, y \in-C \backslash\{0\}$, one has $\left\langle c^{*}, y\right\rangle<0$. Moreover, as $\left\langle c^{*}, c_{n}\right\rangle \geq 0$, one has $\left\langle c^{*}, y_{n}-y_{0}\right\rangle<0$ for sufficiently large $n$. Thus,

$$
\left\langle c^{*}, y_{n}-y_{0}\right\rangle+\left\langle d^{*}, z_{n}\right\rangle<0 .
$$

This is a contradiction to the assumption. Hence, this completes the proof. 


\section{Applications to duality}

Motivated by the work reported in $[1,7,41,42]$, we employ some results obtained in Section 4 to consider some duality schemes for global weak minimizer of the problem $(\mathrm{P})$. In this section, we always consider $x_{0} \in \Omega$, $y_{0} \in F\left(x_{0}\right), z_{0} \in G\left(x_{0}\right) \cap(-D), u \in X,(v, w) \in(F, G)(u)+C \times D$, and assume that $(F, G)$ is $C \times D$-convex and $E_{(F, G)}^{\prime \prime}\left(x-x_{0}\right)$ fulfills the domination property for all $x \in \Omega$.

\subsection{Mond-Weir duality}

We consider a Mond-Weir type dual problem, denoted by $\left(\mathrm{D}_{M W}\right)$, of $(\mathrm{P})$ as follows

maximize $y_{0}$

s.t. $\left\langle c^{*}, y^{\prime}\right\rangle+\left\langle d^{*}, z^{\prime}\right\rangle \geq 0$ for all $\left(y^{\prime}, z^{\prime}\right) \in D_{g}^{\prime \prime}(F, G)\left(x_{0},\left(y_{0}, z_{0}\right), u-x_{0},\left(v-y_{0}, w-z_{0}\right)\right)\left(x-x_{0}\right), x \in \Omega$

$x_{0} \in \Omega, \quad y_{0} \in F\left(x_{0}\right), \quad z_{0} \in G\left(x_{0}\right) \cap(-D)$,

$c^{*} \in C^{*} \backslash\{0\}, \quad d^{*} \in D^{*}, \quad$ and $\left\langle d^{*}, z_{0}\right\rangle=0$.

A point $\left(c^{*}, d^{*}, x_{0}, y_{0}, z_{0}\right)$ is called a feasible solution of $\left(\mathrm{D}_{M W}\right)$ if it satisfies (5.1)-(5.3). Let

$$
\Delta:=\left\{y \in F(x) \mid \text { there exist } c^{*}, d^{*}, z \text { such that }\left(q^{*}, d^{*}, x, y, z\right) \text { satisfies conditions (5.1)-(5.3) }\right\} .
$$

A feasible solution $\left(c^{*}, d^{*}, x_{0}, y_{0}, z_{0}\right)$ is a weak maximizer of $\Delta$ if

$$
\left(\Delta-y_{0}\right) \cap \operatorname{int} C=\emptyset .
$$

Proposition 5.1 (Weak duality). If $(\bar{x}, \bar{y})$ is a feasible solution of $(\mathrm{P})$ and $\left(c^{*}, d^{*}, x_{0}, y_{0}, z_{0}\right)$ is a feasible solution of $\left(\mathrm{D}_{M W}\right)$, then $\left\langle c^{*}, \bar{y}\right\rangle \geq\left\langle c^{*}, y_{0}\right\rangle$.

Proof. Because $(\bar{x}, \bar{y})$ is a feasible solution of $(\mathrm{P})$, then $\bar{x} \in \Omega, \bar{y} \in F(\bar{x})$ and there is $\bar{z} \in G(\bar{x}) \cap(-D)$. It follows from Proposition 3.10 that

$$
(F, G)(\bar{x})-\left(y_{0}, z_{0}\right) \subseteq D_{g}^{\prime \prime}(F, G)\left(x_{0},\left(y_{0}, z_{0}\right), u-x_{0},\left(v-y_{0}, w-z_{0}\right)\right)\left(\bar{x}-x_{0}\right)+C \times D .
$$

Thus, there exit $\left(y^{\prime}, z^{\prime}\right) \in D_{g}^{\prime \prime}(F, G)\left(x_{0},\left(y_{0}, z_{0}\right), u-x_{0},\left(v-y_{0}, w-z_{0}\right)\right)\left(\bar{x}-x_{0}\right)$ and $(c, d) \in C \times D$, such that $\bar{y}-y_{0}=y^{\prime}+c$ and $\bar{z}-z_{0}=z^{\prime}+d$. Moreover, $\left(c^{*}, d^{*}, x_{0}, y_{0}, z_{0}\right)$ is a feasible solution of $\left(\mathrm{D}_{M W}\right)$, one gets

$$
\left\langle c^{*}, \bar{y}-y_{0}\right\rangle+\left\langle d^{*}, \bar{z}-z_{0}\right\rangle=\left\langle c^{*}, y^{\prime}+c\right\rangle+\left\langle d^{*}, z^{\prime}+d\right\rangle=\left\langle c^{*}, y^{\prime}\right\rangle+\left\langle d^{*}, z^{\prime}\right\rangle+\left\langle c^{*}, c\right\rangle+\left\langle d^{*}, d\right\rangle \geq 0 .
$$

As $\bar{z} \in-D$ and $\left\langle d^{*}, z_{0}\right\rangle=0$, one has $\left\langle d^{*}, \bar{z}-z_{0}\right\rangle \leq 0$. It follows form this inequality and (5.3) that $\left\langle c^{*}, \bar{y}-y_{0}\right\rangle \geq 0$, and hence one obtain $\left\langle c^{*}, \bar{y}\right\rangle \geq\left\langle c^{*}, y_{0}\right\rangle$. Thus, the proof is complete.

Proposition 5.2 (Strong duality I). Assume that $(\bar{x}, \bar{y})$ is a feasible solution of $(\mathrm{P}),\left(c^{*}, d^{*}, x_{0}, y_{0}, z_{0}\right)$ is a feasible solution of $\left(\mathrm{D}_{M W}\right)$, and $\left\langle c^{*}, \bar{y}\right\rangle=\left\langle c^{*}, y_{0}\right\rangle$, then $(\bar{x}, \bar{y})$ is a global weak minimizer of $(\mathrm{P})$ and $\left(c^{*}, d^{*}, x_{0}, y_{0}, z_{0}\right)$ is a global weak maximizer of $\left(\mathrm{D}_{M W}\right)$

Proof. - Suppose that $(\bar{x}, \bar{y})$ is not a global weak minimizer of $(\mathrm{P})$, there are $\hat{x} \in \Omega$ and $\hat{y} \in F(\hat{x})$ such that $\hat{y}-\bar{y} \in-\operatorname{int} C$. By $c^{*} \in C^{*} \backslash\{0\},\left\langle c^{*}, \hat{y}\right\rangle<\left\langle c^{*}, \bar{y}\right\rangle=\left\langle c^{*}, y_{0}\right\rangle$, a contradiction to Proposition 5.1. Consequently, $(\bar{x}, \bar{y})$ is a global weak minimizer.

- Now, we assume that $\left(c^{*}, d^{*}, x_{0}, y_{0}, z_{0}\right)$ is not a global weak maximizer of $\left(\mathrm{D}_{M W}\right)$, there exist a feasible solution $\left(\widehat{c^{*}}, \widehat{d^{*}}, x^{\prime}, y^{\prime}, z^{\prime}\right)$ of the problem $\left(\mathrm{D}_{M W}\right)$ such that $y^{\prime}-y_{0} \in \operatorname{int} C$. As $c^{*} \in C^{*} \backslash\{0\}$, we have $\left\langle\widehat{c^{*}}, y^{\prime}\right\rangle>\left\langle\widehat{c^{*}}, y_{0}\right\rangle$. On the other hand, $\left(x_{0}, y_{0}\right)$ is feasible solution of $(\mathrm{P})$ and $\left(\widehat{c^{*}}, \widehat{d^{*}}, x^{\prime}, y^{\prime}, z^{\prime}\right)$ is feasible solution of the problem $\left(\mathrm{D}_{M W}\right)$, according to Proposition 5.1, $\left\langle\widehat{c}^{*}, y_{0}\right\rangle \geq\left\langle\hat{c}^{*}, y^{\prime}\right\rangle$, a contradiction. Consequently, $\left(c^{*}, d^{*}, x_{0}, y_{0}, z_{0}\right)$ is a global weak maximizer of $\left(\mathrm{D}_{M W}\right)$. 
Proposition 5.3 (Strong duality II). If $\left(x_{0}, y_{0}\right)$ is a global weak minimizer of (P) and the (KRZCQ) in Theorem 4.2 holds, then there are $c^{*} \in C^{*} \backslash\{0\}$ and $d^{*} \in D^{*}$ such that $\left(c^{*}, d^{*}, x_{0}, y_{0}, z_{0}\right)$ is a global weak maximizer of $\left(D_{M W}\right)$.

Proof. It follows from Theorem 4.2 that there are $c^{*} \in C^{*} \backslash\{0\}$ and $d^{*} \in D^{*}$ such that $\left(c^{*}, d^{*}, x_{0}, y_{0}, z_{0}\right)$ is a feasible solution of $\left(\mathrm{D}_{M W}\right)$. By the same arguments as in Proposition 5.3, we conclude that $\left(c^{*}, d^{*}, x_{0}, y_{0}, z_{0}\right)$ is a global weak maximizer of $\left(\mathrm{D}_{M W}\right)$ and this completes the proof.

Next we employ Theorem 4.11 to write down the following proposition.

Proposition 5.4. (Converse duality) If $\left(c^{*}, d^{*}, x_{0}, y_{0}, z_{0}\right)$ is a feasible solution of $\left(\mathrm{D}_{M W}\right)$ then $\left(x_{0}, y_{0}\right)$ is a global weak minimizer of $(\mathrm{P})$.

\subsection{Wolfe duality}

We define the Wolfe dual problem $\left(\mathrm{D}_{W}\right)$ as follows

$$
\begin{aligned}
& \operatorname{maximize} \quad \psi\left(c^{*}, d^{*}, x_{0}, y_{0}, z_{0}\right)=\left\langle q^{*}, y_{0}\right\rangle+\left\langle d^{*}, z_{0}\right\rangle \\
& \text { s.t. }\left\langle c^{*}, y^{\prime}\right\rangle+\left\langle d^{*}, z^{\prime}\right\rangle \geq 0 \text { for all }\left(y^{\prime}, z^{\prime}\right) \in D_{g}^{\prime \prime}(F, G)\left(x_{0},\left(y_{0}, z_{0}\right), u-x_{0},\left(v-y_{0}, w-z_{0}\right)\right)\left(x-x_{0}\right), x \in \Omega \\
& x_{0} \in \Omega, \quad y_{0} \in F\left(x_{0}\right), \quad z_{0} \in G\left(x_{0}\right) \cap(-D), \\
& c^{*} \in C^{*} \backslash\{0\}, \quad d^{*} \in D^{*} .
\end{aligned}
$$

A point $\left(c^{*}, d^{*}, x_{0}, y_{0}, z_{0}\right)$ is a feasible solution of $(\mathrm{D})_{W}$ if it satisfies $(5.5)-(5.7) .\left(c^{*}, d^{*}, x_{0}, y_{0}, z_{0}\right)$ is an optimal solution of $(\mathrm{D})_{W}$ if for any feasible solution $\left(c^{* *}, d^{*}, x^{\prime}, y^{\prime}, z^{\prime}\right)$,

$$
\psi\left(c^{*}, d^{*}, x_{0}, y_{0}, z_{0}\right) \geq \psi\left(c^{\prime *}, d^{\prime *}, x^{\prime}, y^{\prime}, z^{\prime}\right) .
$$

Proposition 5.5 (Weak duality). If $(\bar{x}, \bar{y})$ is a feasible solution of $(\mathrm{P})$ and $\left(c^{*}, d^{*}, x_{0}, y_{0}, z_{0}\right)$ is a feasible solution of $\left(\mathrm{D}_{W}\right)$, then $\left\langle c^{*}, \bar{y}\right\rangle \geq \psi\left(c^{*}, d^{*}, x_{0}, y_{0}, z_{0}\right)$.

Proof. By virtue of Proposition 3.10, one has

$$
(F, G)(\bar{x})-\left(y_{0}, z_{0}\right) \subseteq D_{g}^{\prime \prime}(F, G)\left(x_{0},\left(y_{0}, z_{0}\right), u-x_{0},\left(v-y_{0}, w-z_{0}\right)\right)\left(\bar{x}-x_{0}\right)+C \times D .
$$

Hence, there exit $\left(y^{\prime}, z^{\prime}\right) \in D_{g}^{\prime \prime}(F, G)\left(x_{0},\left(y_{0}, z_{0}\right), u-x_{0},\left(v-y_{0}, w-z_{0}\right)\right)\left(\bar{x}-x_{0}\right)$ and $(c, d) \in C \times D$, such that $\bar{y}-y_{0}=y^{\prime}+c$ and $\bar{z}-z_{0}=z^{\prime}+d$. Since $\left(c^{*}, d^{*}, x_{0}, y_{0}, z_{0}\right)$ is a feasible solution of $\left(\mathrm{D}_{W}\right)$, one gets

$$
\left\langle c^{*}, \bar{y}-y_{0}\right\rangle+\left\langle d^{*}, \bar{z}-z_{0}\right\rangle=\left\langle c^{*}, y^{\prime}+c\right\rangle+\left\langle d^{*}, z^{\prime}+d\right\rangle=\left\langle c^{*}, y^{\prime}\right\rangle+\left\langle d^{*}, z^{\prime}\right\rangle+\left\langle c^{*}, c\right\rangle+\left\langle d^{*}, d\right\rangle \geq 0 .
$$

As $\bar{z} \in-D,\left\langle c^{*}, \bar{y}\right\rangle \geq\left\langle q^{*}, y_{0}\right\rangle+\left\langle d^{*}, z_{0}\right\rangle-\left\langle d^{*}, d\right\rangle \geq\left\langle q^{*}, y_{0}\right\rangle+\left\langle d^{*}, z_{0}\right\rangle$. Hence, we get that

$$
\left\langle c^{*}, \bar{y}\right\rangle \geq \psi\left(c^{*}, d^{*}, x_{0}, y_{0}, z_{0}\right) \text {. }
$$

Proposition 5.6 (Strong duality). If $\left(x_{0}, y_{0}\right)$ is a global weak minimizer of $(\mathrm{P})$, where $y_{0}=0$, and the (KRZCQ) in Theorem 4.2 holds, then there are $c^{*} \in C^{*} \backslash\{0\}$ and $d^{*} \in D^{*}$ such that $\left(c^{*}, d^{*}, x_{0}, y_{0}, z_{0}\right)$ is an optimal solution of of $\left(D_{W}\right)$.

Proof. By Theorem 4.2, there are $c^{*} \in C^{*} \backslash\{0\}$ and $d^{*} \in D^{*}$ with $\left\langle d^{*}, z_{0}\right\rangle=0$ such that $\left(c^{*}, d^{*}, x_{0}, y_{0}, z_{0}\right)$ is a feasible solution of $\left(\mathrm{D}_{W}\right)$. Assume that $\left(c^{*}, d^{*}, x_{0}, y_{0}, z_{0}\right)$ is not an optimal solution of $\left(\mathrm{D}_{W}\right)$. Then, there exists a feasible solution $\left(c^{\prime *}, d^{\prime *}, x^{\prime}, y^{\prime}, z^{\prime}\right)$ of $\left(\mathrm{D}_{W}\right)$ such that

$$
\psi\left(c^{*}, d^{*}, x_{0}, y_{0}, z_{0}\right)<\psi\left(c^{\prime *}, d^{\prime *}, x^{\prime}, y^{\prime}, z^{\prime}\right) .
$$

As $\left(x_{0}, y_{0}\right)$ is a feasible of $(\mathrm{P})$, by Proposition 5.5, one has $\psi\left(c^{\prime *}, d^{\prime *}, x^{\prime}, y^{\prime}, z^{\prime}\right) \leq\left\langle c^{\prime *}, y_{0}\right\rangle$. Moreover, $\psi\left(c^{*}, d^{*}, x_{0}, y_{0}, z_{0}\right)=\left\langle c^{*}, y_{0}\right\rangle$, then $\left\langle c^{*}, y_{0}\right\rangle\left\langle\left\langle c^{* *}, y_{0}\right\rangle\right.$, meaning that $\left\langle c^{*}-c^{\prime *}, 0\right\rangle<0$, a contradiction to $y_{0}=0$. The proof is complete. 
Finally we apply Theorem 4.11 to obtain the following result of converse duality.

Proposition 5.7 (Converse duality). If $\left(c^{*}, d^{*}, x_{0}, y_{0}, z_{0}\right)$ is a feasible solution of $\left(\mathrm{D}_{W}\right)$ then $\left(x_{0}, y_{0}\right)$ is a global weak minimizer of $(\mathrm{P})$.

Acknowledgements. The author is grateful to the anonymous referees for their valuable suggestions and remarks which have greatly improved the first version of the paper. A part of the work was completed during a stay of the authors at the Vietnam Institute for Advanced Study in Mathematics (VIASM). The author would like to thank VIASM for its hospitality and support. This work was supported by the National Foundation for Science and Technology Development (NAFOSTED) grand 101.01-2020.23.

\section{REFERENCES}

[1] N.L.H. Anh, Higher-order optimality conditions in set-valued optimization using Studniarski derivatives and applications to duality. Positivity 18 (2014) 449-473.

[2] J.P. Aubin, Contingent Derivatives of Set-Valued Maps and Existence of Solutions to Nonlinear Inclusions and Differential Inclusions, Mathematical Analysis and Applications, Part A, edited by L. Nachbin. Academic Press, New York (1981) 160-229.

[3] J.P. Aubin and H. Frankowska, Set-Valued Analysis. Birkhauser, Boston (1990).

[4] H.P. Benson, An improved definition of proper efficiency for vector minimization with respect to cones. J. Math. Anal. Appl. 71 (1979) 232-241.

[5] J.M. Bonnisseau and B. Cornet, Existence of equilibria when firms follow bounded losses pricing rules. J. Math. Econ. 17 (1988) 119-147.

[6] G.Y. Chen and J. Jahn, Optimality conditions for set-valued optimization problems. Math. Methods Oper. Res. 48 (1988) 187-200.

[7] C.R. Chen, S.J. Li and K.L. Teo, Higher order weak epiderivatives and applications to duality and optimality conditions. Comput. Math. Appl. 57 (2009) 1389-1399.

[8] R. Cominetti, Metric regularity, tangent sets, and second-order optimality conditions. Appl. Math. Optim. 21 (1990) $265-287$.

[9] H.W. Corley, Optimality conditions for maximizations of set-valued functions. J. Optim. Theory App. 58 (1988) 1-10.

[10] A.L. Dontchev and R.T. Rockafellar, Implicit Functions and Solution Mappings. Springer, Berlin (2009).

[11] M. Durea, Optimality conditions for weak and firm efficiency in set-valued optimization. J. Math. Anal. Appl. 344 (2008) 1018-1028.

[12] A. Götz and J. Jahn, The Lagrange multiplier rule in set-valued optimization. SIAM J. Optim. 10 (1999) 331-344.

[13] C. Gutiérrez, B. Jiménez and V. Novo, On second order Fritz John type optimality conditions in nonsmooth multiobjective programming. Math. Program. Ser. B. 123 (2010) 199-223.

[14] M.I. Henig, Proper efficiency with respect to cones. J. Optim. Theory App. 36 (1982) 387-407.

[15] G. Isac and A.A. Khan, Dubovitskii-Milyutin approach in set-valued optimization. SIAM J. Control Optim. 47 (2008) $144-162$.

[16] J. Jahn, Vector Optimization: Theory, Applications, and Extensions. Springer, Berlin (2004).

[17] J. Jahn and A.A. Khan, Generalized contingent epiderivatives in set-valued optimization: optimality conditions. Numer. Funct. Anal. Optim. 23 (2002) 807-831.

[18] J. Jahn and R. Rauh, Contingent epiderivative and set-valued optimization. Math. Methods Oper. Res. 46 (1997) 193-211.

[19] J. Jahn, A.A. Khan and P. Zeilinger, Second-order optimality conditions in set optimization. J. Optim. Theory App. 125 (2005) 331-347.

[20] B. Jiménez and V. Novo, Second-order necessary conditions in set constrained differentiable vector optimization. Math. Methods Oper. Res. 58 (2003) 299-317.

[21] B. Jiménez and V. Novo, Optimality conditions in differentiable vector optimization via second-order tangent sets. Appl. Math. Optim. 49 (2004) 123-144.

[22] A. Jofré and A. Jourani, Characterizations of the free disposal condition for nonconvex economies on infinite dimensional commodity spaces. SIAM J. Optim. 25 (2015) 699-712.

[23] A. Jofré and J. Rivera, An intrinsic characterization of free disposal hypothesis. Econ. Lett. 92 (2006) $423-427$.

[24] H. Kawasaki, An envelope-like effect of infinitely many inequality constraints on second order necessary conditions for minimization problems. Math. Program. 41 (1988) 73-96.

[25] A.A. Khan and C. Tammer, Second-order optimality conditions in set-valued optimization via asymptotic derivatives. Optimization 62 (2013) 743-758.

[26] P.Q. Khanh and N.D. Tuan, Second order optimality conditions with the envelope-like effect in nonsmooth multiobjective programming II: optimality conditions. J. Math. Anal. Appl. 403 (2013) 703-714.

[27] P.Q. Khanh and N.D. Tuan, Second-order optimality conditions with the envelope-like effect for nonsmooth vector optimization in infinite dimensions. Nonlinear Anal. 77 (2013) 130-148.

[28] P.Q. Khanh and N.M. Tung, Second-order optimality conditions with the envelope-like effect for set-valued optimization. J. Optim. Theory App. 167 (2015) 68-90. 
[29] P.Q. Khanh and N.M. Tung, Existence and boundedness of second-order Karush-Kuhn-Tucker multipliers for set-vlued optimization with variable ordering structures, Taiwan. J. Math. 22 (2018) 1001-1029.

[30] P.Q. Khanh and N.M. Tung, Higher-order Karush-Kuhn-Tucker conditions in nonsmooth optimization. SIAM J. Optim. 28 (2018) 820-848.

[31] S.J. Li, K.L. Teo and X.Q. Yang, Higher-order Mond-Weir duality for set-valued optimization. J. Comput. Appl. Math. 217 (2008) 339-349.

[32] S.J. Li, S.K. Zhu and K.L. Teo, New generalized second-order contingent epiderivatives and set-valued optimization problems. J. Optim. Theory App. 152 (2012) 587-604.

[33] D.T. Luc, Theory of Vector Optimization. Springer, Berlin (1989).

[34] B.S. Mordukhovich, Variational Analysis and Generalized Differentiation, Vol. I Basic Theory, Vol. II Applications. Springer, Berlin (2006).

[35] J.P. Penot, Second order conditions for optimization problems with constraints. SIAM J. Control Optim. 37 (1999) $303-318$.

[36] S.M. Robinson, Regularity and stability for convex multivalued functions. Math. Oper. Res. 1 (1976) $130-143$.

[37] P.H. Sach, N.D. Yen and B.D. Craven, Generalized invexity and duality theorems with multifunctions. Numer. Funct. Anal. Optim. 15 (1994) 131-153.

[38] X.K. Sun and S.J. Li, Lower Studniarski derivative of the perturbation map in parametrized vector optimization. Optim Lett. 5 (2011) 601-614.

[39] X.K. Sun and S.J. Li, Generalized second-order contingent epiderivatives in parametric vector optimization problems. J. Global Optim. 58 (2014) 351-363.

[40] X.K. Sun, Z.Y. Peng and X.L. Guo, Some characterizations of robust optimal solutions for uncertain convex optimization problems. Optim Lett. 10 (2016) 1463-1478.

[41] X.K. Sun, K.L. Teo and L.P. Tang, Dual approaches to characterize robust optimal solution sets for a class of uncertain optimization problems. J. Optim. Theory App. 182 (2019) 984-1000.

[42] X.K. Sun, K.L. Teo, J. Zeng and L.Y. Liu, Robust approximate optimal solutions for nonlinear semi-infinite programming with uncertainty. Optimization 69 (2020) 2109-2129.

[43] C. Ursescu, Multifunctions with closed convex graph. Czech. Math. J. 25 (1975) 438-441.

[44] T. Weir and B. Mond, Generalized convexity and duality in multiple objective programming. Bull. Aust. Math. Soc. 39 (1989) $287-299$.

[45] X.Y. Zheng and K.F. Ng, The Fermat rule for multifunctions on Banach spaces. Math. Program. Ser. A 104 (2005) 69-90.

[46] S.K. Zhu, S.J. Li and K.L. Teo, Second-order Karush-Kuhn-Tucker optimality conditions for set-valued optimization. J. Global Optim. 58 (2014) 673-679.

[47] J. Zowe and S. Kurcyusz, Regularity and stability for the mathematical programming problem in Banach spaces. Appl. Math. Optim. 5 (1979) 49-62. 